\title{
Parametric Geometry Models for Hypersonic Aircraft Components: Blunt Leading Edges
}

\author{
Konstantinos Kontogiannis \\ University of Southampton \\ András Sóbester** \\ University of Southampton
}

\author{
Adriano Cerminara* \\ University of Southampton \\ Neil Sandham ${ }^{\dagger \dagger}$ \\ University of Southampton
}

Nigel Taylor ${ }^{\S}$

$M B D A U K$

In this paper we report the results of investigations into the efficient parameterization of blunt leading edge shapes for hypersonic aircraft geometries. The investigations mostly revolve around waverider geometries generated with inverse design techniques, such as the osculating cones waverider forebody design method. The shapes presented however, can be utilized to introduce bluntness to any wedge-like geometry with sharp leading edges. Initially, we present detailed descriptions of three different variations of the rational Bézier curve based parameterization that was developed, and the variety of shapes that can be obtained is demonstrated. Afterwards their performance is evaluated utilizing 2D CFD analysis. In our simulations, the rational Bézier curve leading edges outperform circular ones when it comes to minimizing both drag and peak heating rates or peak temperatures. Additionally, with higher order rational Bézier leading edge shapes, higher levels of geometric continuity can be achieved at the interface between the blunt part and the original wedge-like geometry, resulting in a smoother transition. Preliminary results indicate that this can potentially affect the receptivity and hence transition mechanisms. Finally, the 2D geometry formulations are extended to full 3D waverider forebody geometries.

\begin{tabular}{|c|c|c|c|}
\hline \multicolumn{4}{|c|}{ Nomenclature } \\
\hline $\mathrm{C}_{\mathrm{d}}$ & $=$ drag coefficient & $\mathrm{R}$ & $=$ circular leading edge radius \\
\hline CFD & $=$ Computational Fluid Dynamics & RBLE & $=$ rational Bézier leading edge \\
\hline $\mathrm{d}$ & $=$ local blunt section thickness & $\mathrm{t}$ & $=$ parametric curve parameter $[0,1]$ \\
\hline $\mathrm{k}$ & $=$ thermal conductivity & $\mathrm{U}_{\mathrm{inf}}$ & $=$ freestream velocity \\
\hline $\mathrm{M}_{\mathrm{inf}}$ & $=$ freestream Mach number & $\operatorname{var}_{i}$ & $=$ design variable \\
\hline $\mathrm{n}$ & $=$ Bézier curve order & $\mathrm{W}_{\mathrm{i}}$ & $=$ rational Bézier weight \\
\hline $\mathrm{P}_{\mathrm{i}}$ & $=$ parametric curve control point & & \\
\hline$p_{\text {inf }}$ & $=$ freestream pressure & $\varepsilon$ & $=$ wall emissivity \\
\hline $\mathrm{p}_{\mathrm{w}}^{\prime}$ & $=$ wall pressure fluctuation amplitude & $\lambda$ & $=$ local sweep angle \\
\hline $\mathrm{p}_{\text {inf }}^{\prime}$ & $=$ freestream pressure fluctuation amplitude & $\mu_{\text {inf }}$ & $=$ freestream dynamic viscosity \\
\hline q & $=$ wall heat flux & $\rho$ & $=$ local radius of curvature \\
\hline $\mathrm{q}^{\prime}{ }_{\mathrm{w}}$ & $=$ heat flux fluctuation amplitude & $\rho_{\text {inf }}$ & $=$ freestream density \\
\hline
\end{tabular}

\section{Introduction}

$\mathrm{W}$ AVERIDERS are a class of aircraft geometries that keep the shockwave generated by the oncoming hypersonic flow attached to their leading edge. The result of this is superior lift values when compared to conventional designs, rendering waveriders one of the most promising configurations for hypersonic flight. There are a number of inverse design methods to generate waverider geometries, from very simple ones to much more

\footnotetext{
$\dagger$ Postgraduate Research Student, University of Southampton, Boldrewood Campus, Southampton, UK, SO16 7QF.

* Postgraduate Research Student, University of Southampton, Highfield, Southampton, UK, SO17 1BJ.

$\S$ Capability Leader Aerodynamic Tools \& Methods, MBDA, PO Box 5, Filton, UK, BS34 7QW; SMAIAA.

** Associate Professor, University of Southampton, Boldrewood Campus, Southampton, UK, SO16 7QF; SMAIAA.

${ }^{\dagger}$ Professor, University of Southampton, Highfield Campus, Southampton, UK, SO17 1BJ.
} 
sophisticated and flexible ones ${ }^{1-4}$, that have the advantage of being computationally cheap while based on inviscid flowfields. These methods however are only able to provide a baseline shape that needs to be further adapted and refined in order to obtain more realistic aircraft geometries. Additionally, a distinctive characteristic of hypersonic aircraft designs is the level of integration between the airframe and propulsion system, making optimization studies more complex and emphasizing the need for efficiently parameterized geometry models.

One of the first issues that need to be addressed when departing from the simplicity of inviscid designs is that of the aerodynamic heating effects that, along with manufacturability constraints, preclude the use of sharp leading edges at hypersonic speeds. Two early examples of non-ballistic high-speed vehicle geometries that needed blunt leading edges are the space shuttle and the X-15 research plane. Waverider shapes, however, rely on the sharpness of their leading edge in order to keep the shock attached to it, and for this reason the performance of such aircraft is more sensitive and is expected to degrade with the use of finite radius leading edges. It is, therefore, of great interest to investigate methods to optimally introduce bluntness to the leading edge of such vehicles.

Over the years, a number of approaches to introducing bluntness to leading edges of slender bodies have been proposed. Most common are circular leading edge shapes that have been widely used and studied due to their simplicity. There are however benefits in looking into more sophisticated shapes than a constant radius of curvature for the leading edge bluntness, and a number of studies have looked into different geometry parameterizations for this purpose. Power-law shapes have been considered in a number of studies that explored potential advantages of having more control over the shape of the blunted part ${ }^{5-6}$. More recently, parametric curves able to achieve a wide variety of shapes have also been considered with promising results ${ }^{7-8}$.

The aim of this work is to devise efficiently parameterized geometries for hypersonic aircraft components. In Ref. 9 we briefly presented a parametric geometry model for introducing bluntness to the leading edge of waverider forebodies. It is a geometric formulation able to achieve a wide variety of shapes with desirable characteristics, while keeping the number of associated design variables low. Here, we will have a more detailed look into those rational Bézier curve based leading edge (RBLE) shapes, starting from their purely geometrical characteristics and how to efficiently control them, to evaluating their aero-thermal performance characteristics. This will be backed up with CFD analyses of the geometries, where their performance is also compared to the more common circular leading edges. We will also present some preliminary results on receptivity and turbulent transition mechanism investigations conducted to examine the effect of the increased geometric continuity of the proposed geometries. Finally, we show a method to integrate these 2D shapes onto 3D waverider forebody geometries.

\section{Leading Edge Geometry Parameterization}

Sharp leading edges of waverider forebodies can generally be described as wedge-like geometries on the vehicle's cross sections. Such section profiles allow for a parametric curve as simple as a quadratic Bézier curve to be used to generate a curved blunt leading edge shape after truncating the original geometry. Using a quadratic Bézier curve, however, fixes the shape of the leading edge, rendering it non-parametric. The two end points would be placed at the edge of the truncated part, on the original geometry, and the intermediate one is restricted in order to satisfy first order continuity at the interfaces of the curve and the original geometry. Two flexible alternatives to this approach can be either using higher order curves with more movable control points, as is the case in Rodi's work ${ }^{7}$, or by using rational Bézier curves whose shape can be further adjusted with weights. The latter of the two approaches has been followed in this work and this is what we discuss next.

\section{A. Quadratic rational Bézier leading edge.}

A quadratic RBLE is the simplest one that can be used. As seen in Figure 1, the original wedge-like geometry is truncated and replaced with a quadratic rational Bézier curve. The parametric curve's end points $\left(\mathrm{P}_{0}\right.$ and $\left.\mathrm{P}_{2}\right)$ are on the interface with the original truncated geometry, while the middle control point $\left(\mathrm{P}_{1}\right)$ is essentially fixed to satisfy continuity conditions at the interfaces since the curve is tangent to its control polygon at its end points. Varying the weight $\mathrm{w}_{1}$ of the middle point then controls the shape, while the weights of the end points, $\mathrm{w}_{0}$ and $\mathrm{w}_{2}$, are fixed at a value of 1 .

Rational Bézier curves are described by:

$$
B(t)=\frac{\sum_{i=0}^{n}\left(\begin{array}{l}
n \\
i
\end{array}\right)(1-t)^{n-i} t^{i} w_{i} P_{i}}{\sum_{i=0}^{n}\left(\begin{array}{l}
n \\
i
\end{array}\right)(1-t)^{n-i} t^{i} w_{i}}
$$




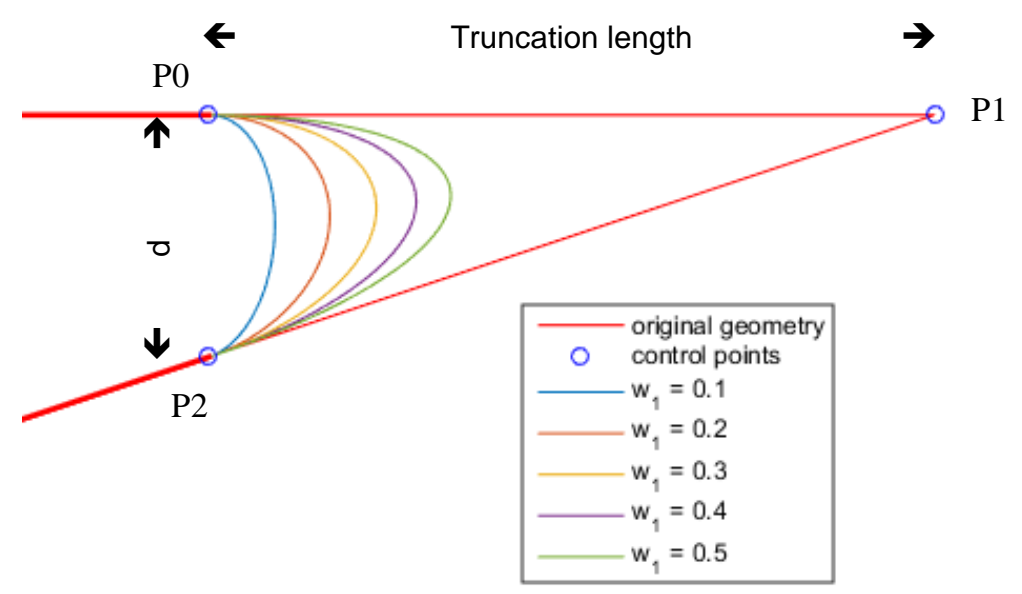

Figure 1. Rational quadratic Bézier curve applied to a 2D wedge-like sharp leading edge with different weights $w_{l}$.

The geometries that are expected to be of most interest and have aero-thermal characteristics that outperform the circular arc leading edges are those that have a larger radius of curvature at the stagnation point and tighter ones closer to the edges, as this better distributes the heat load across the blunted part. An example of such a shape is the bluntest of the family shown in Figure 1 with a $\mathrm{w}_{1}$ value of around 0.1.

These shapes can be controlled with two parameters, the truncation length (or the thickness at the interface) and the weight of the middle point $\mathrm{w}_{1}$. The first can be a design variable itself since it directly controls the scale of the blunted region. However, varying the weight directly produces what could be called a non-linear variation of shapes for the range of values of $\mathrm{w}_{1}$. This is best illustrated in Figure 2, where the weight is directly linked to the design variable that is ranging from 0 to 1 in the first case, and equal to the square of the design variable's value in the second case. The second method offers a more intuitive variation of shapes across the design variable range, with slightly increased resolution at low values where geometries with better aero-thermal characteristics are expected.

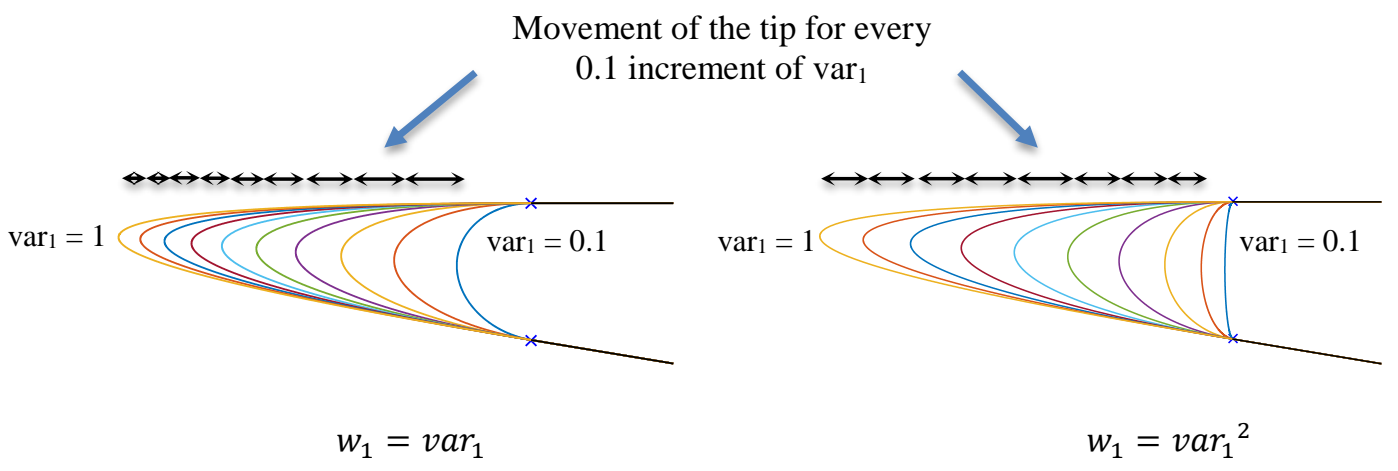

Figure 2. Leading edge shape range for $\mathrm{w}_{1}$ directly linked a design variable (left) and linked to the square of a design variable (right) ranging from 0.1 to 1.0 with equal spacing.

\section{B. Cubic rational Bézier leading edge.}

With the quadratic RBLE shapes, a variety of what intuitively seems like meaningful shapes can be obtained. There could however be benefits in having more control over the shape of the blunted geometry by using parameterizations that provide more flexibility. A simple and elegant way to accomplish this without adding too much complexity with movable control points, is by duplicating the intermediate point. This way the order of the curve increases by one and the two intermediate control points are on top of each other. They are still fixed to satisfy continuity conditions but there are now two weights that can be controlled. The parameterization can be seen in Figure 3. 


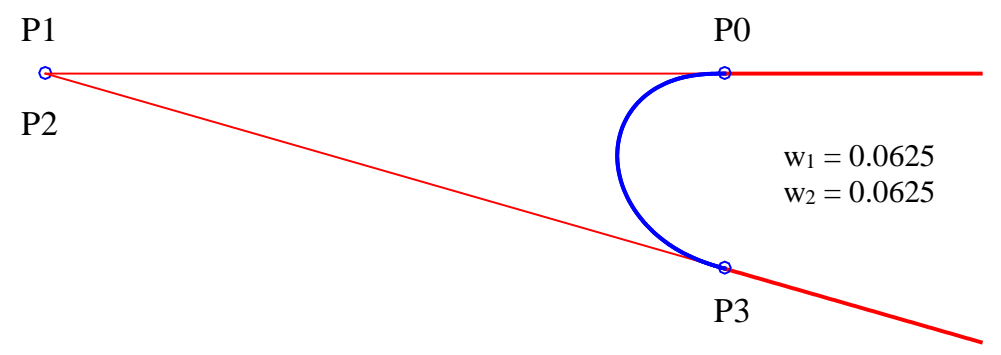

Figure 3. Cubic rational Bézier curve leading edge.

Once again, for more intuitive control, we implement a non-linear mapping between the weights and the design variables. The square of the first variable defines the weights' mean value and the second one is used to balance them.

$$
\begin{aligned}
& w_{1}=\operatorname{var}_{1}^{2}\left(1+\operatorname{var}_{2}\right) \\
& w_{2}=\operatorname{var}_{1}^{2}\left(1-\operatorname{var}_{2}\right)
\end{aligned}
$$

with the variable ranges: $\operatorname{var}_{1} \in(0,1], \operatorname{var}_{2} \in[-1,1]$.

The mean value of the two weights is therefore equal to the square of the first design variable and mostly has control over how far the 'tip' will extend. The second design variable balances the two weights without essentially moving the tip of the geometry in the direction of the flow, but shifts the balance of the shape upwards or downwards. This is best demonstrated by the example geometries that are given in Figure 4 for varying values of the design variables.
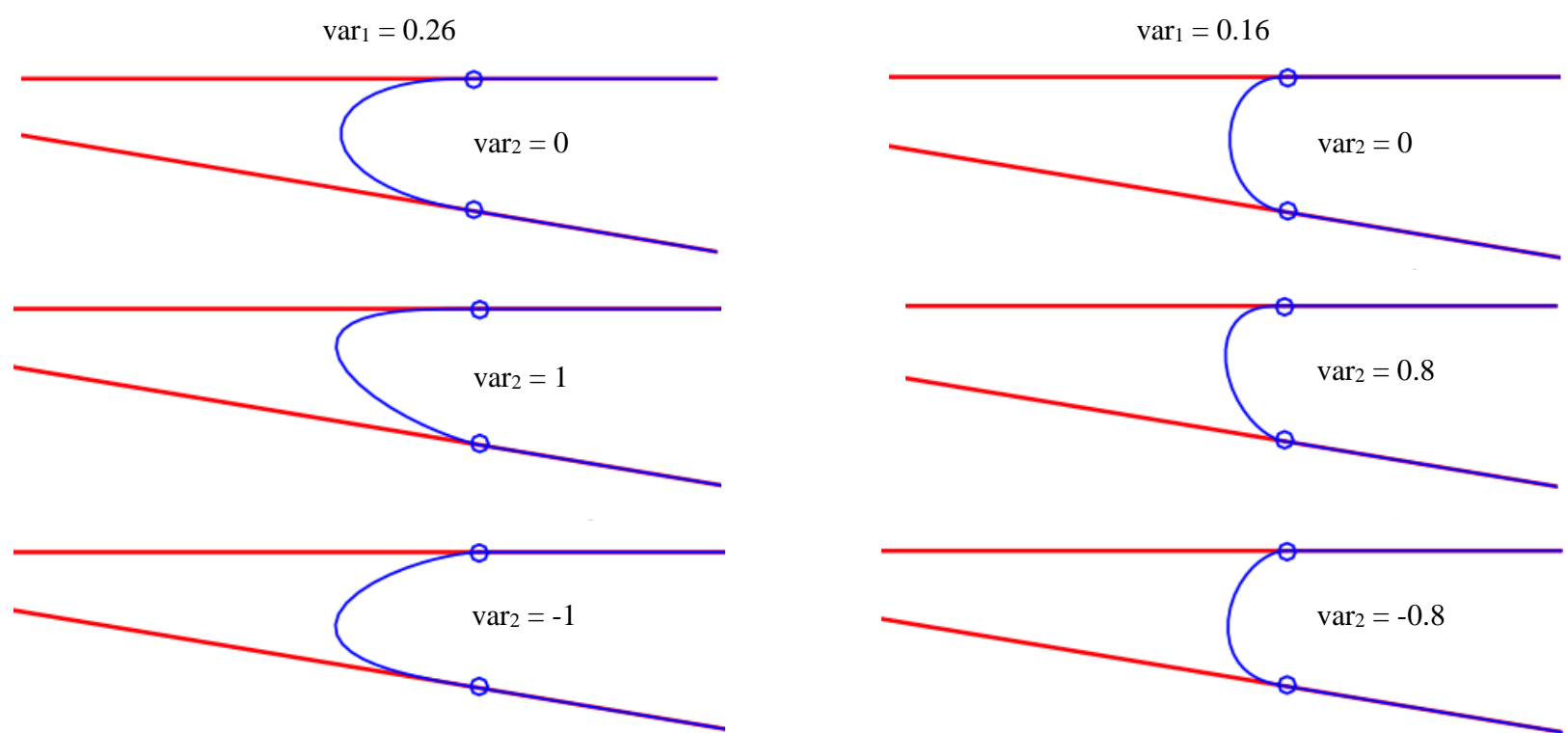

Figure 4. Leading edge shape examples of the cubic rational Bézier parameterization.

Apart from the increased level of control over the shape compared to the first scheme we described, another advantage is the increased level of geometric continuity that can be achieved at the interface between the original wedge-like geometry and the parametric curve. Almost all the approaches that have been considered so far, from the simple circular leading edge shapes, some power law shapes and even parametric curve shapes such as the quadratic RBLE described earlier, only achieve first order geometric continuity $\left(\mathrm{G}^{1}\right)$ at the interface of the blunted part. To the 
human eye this seems smooth enough. However, the flow properties may be sensitive to the jump in the radius of curvature of the surface, potentially affecting the receptivity and turbulent transition mechanisms. The cubic RBLE shapes that are presented here, however, can achieve continuity of the radius of curvature $\left(G^{2}\right)$ at the interface of the blunted part.

The radius of curvature at the endpoints of a rational Bézier curve can be calculated as follows ${ }^{10}$ :

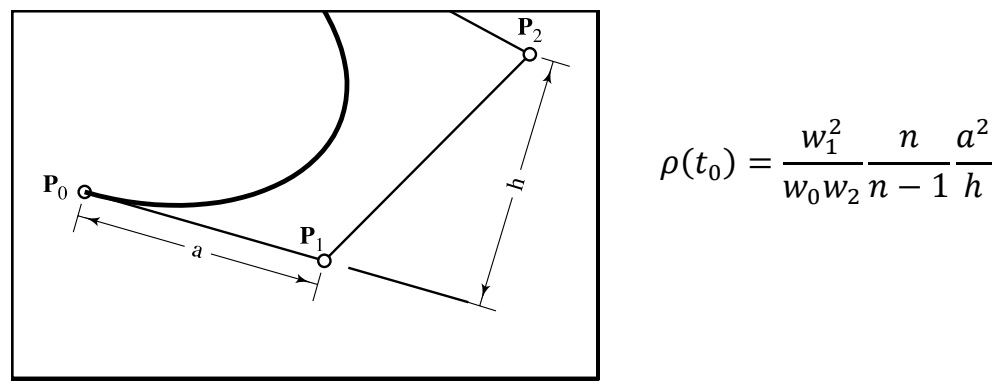

When the initial sharp leading edge consists of straight lines of infinite radius of curvature, the parametric shape described above matches that infinite radius of curvature at the end points, as $h=0$ for $t_{0}$ and $t_{1}$. The numerically calculated radius of curvature at stations along the curve of such a leading edge geometry is shown in Figure 5.
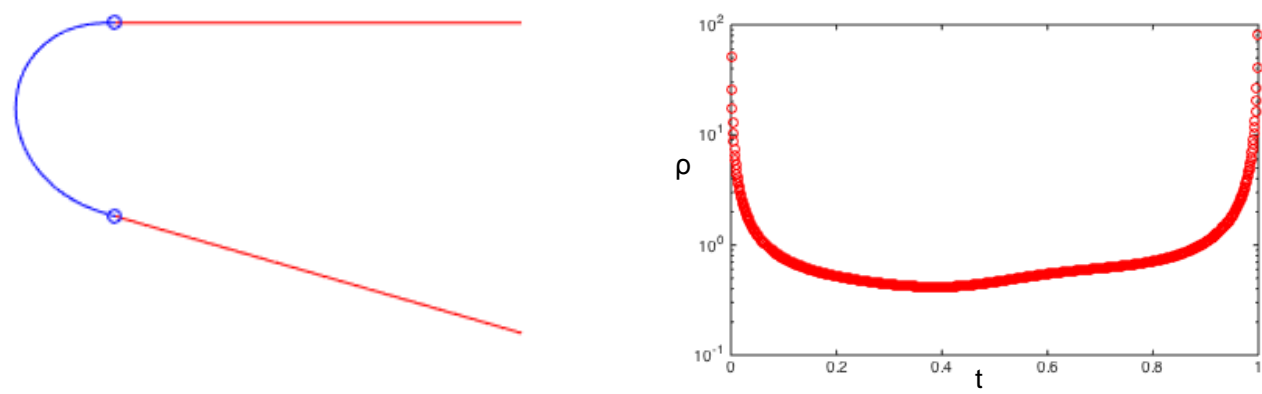

Figure 5. Radius of curvature along cubic rational Bézier leading edge.

It is also possible to match relatively large (compared to the thickness of the blunted region) radii of curvature of the original geometry at the interface, even when the radius of curvature is finite. That usually is the case for the lower surface of waverider geometries or after morphing and further shaping of the upper surface. If, for example, the radius of curvature is finite only on the lower surface, one of the control points can be moved to a position with $h \neq 0$ with respect to one end of the curve. An example is shown in Figure 6, where P2 can essentially move along the line defined by P1 and P3 to obtain the desired radius of curvature at P0 while maintaining an infinite radius of curvature at P3. The parameterization at this point becomes more complex as the control point needs to move according to the values of the weights to keep the radius of curvature constant.
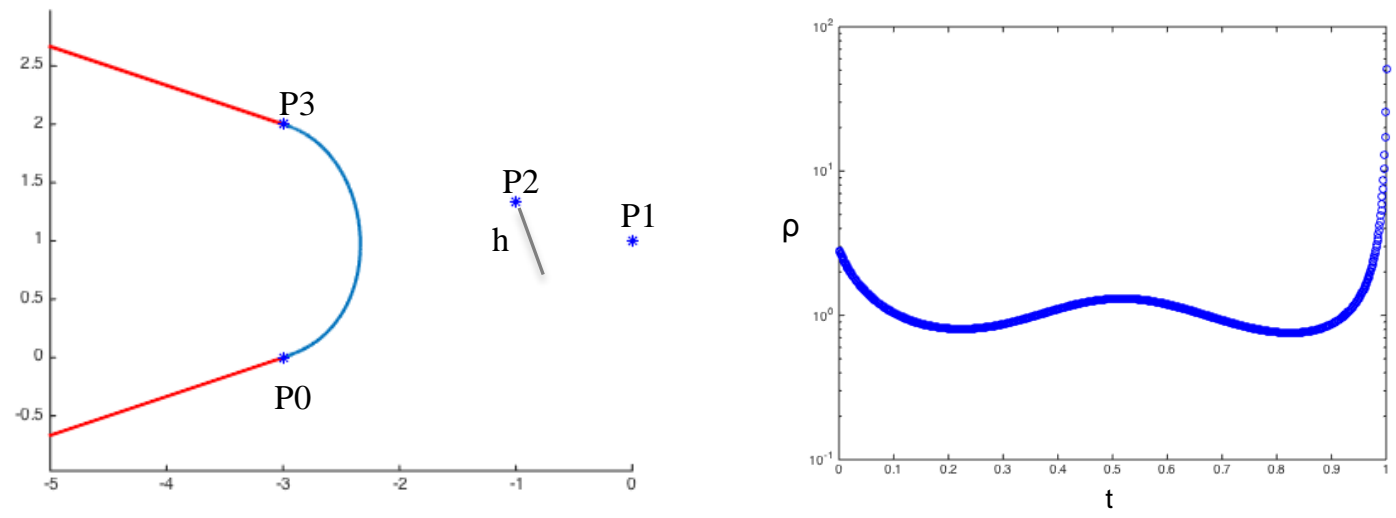

Figure 6. Cubic rational Bézier leading edge with a finite radius of curvature on one end (P0).

American Institute of Aeronautics and Astronautics 


\section{Fourth order rational Bézier leading edge.}

To achieve even greater flexibility, a third intermediate control point can also be placed on top of the two of the previous method. The order of the curve is now four, and the number of adjustable weights for the parameterization increases to three. The addition of a third adjustable weight opens up a wider range of potential shapes that can now be generated. The weights $\mathrm{w}_{1}, \mathrm{w}_{2}$ and $\mathrm{w}_{3}$, control the top, middle and lower part of the shape respectively (when the control points are ordered as seen in Figure 7). Balancing them to obtain meaningful shapes for a range of design variable values, though, is a little more delicate than for the previous two approaches. The relationships between the design variables and weights were chosen here as follows:

$$
\begin{aligned}
w_{1} & =\operatorname{var}_{1}^{2}\left(1+\frac{1}{3} \operatorname{var}_{2}\right) \\
w_{3} & =\operatorname{var}_{1}{ }^{2}\left(1-\frac{1}{3} \operatorname{var}_{2}\right) \\
w_{2} & =-\operatorname{var}_{1}{ }^{2}+0.1 \operatorname{var}_{3}
\end{aligned}
$$

with the variable ranges: $\operatorname{var}_{1} \in(0,1]$, var $_{2} \in[-1,1]$, var $_{3} \in[0,1]$.

P1

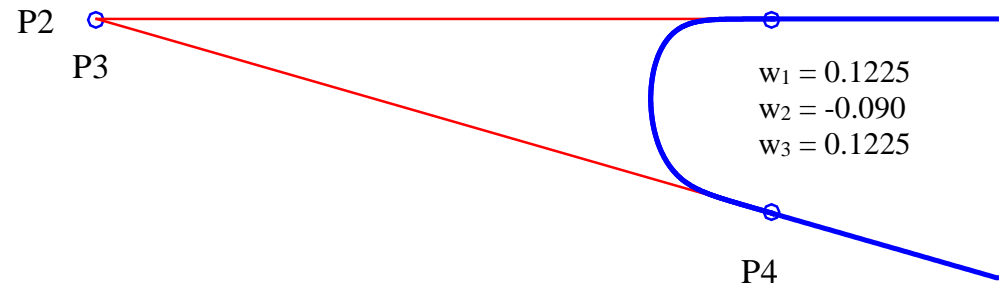

Figure 7. Fourth order rational Bézier curve leading edge.

With the above reparameterization, each of the variables is given a more specific role. The first and the second ones, similar to the cubic RBLE case, control the mean value of the weights at the sides, $\mathrm{w}_{1}$ and $\mathrm{w}_{3}$, and the balance between the two respectively. The weight of the middle point $\mathrm{w}_{2}$, controls how blunt the middle part of the shape is, with its lowest value being roughly the limit where the geometry starts becoming concave. The end result is a shape parameterization where each design variable has a specific role and the characteristics it gives to the final geometry are retained when the other design variables are altered. This is best illustrated in Figure 8, where a number of example geometries are given for different values of the design variables.

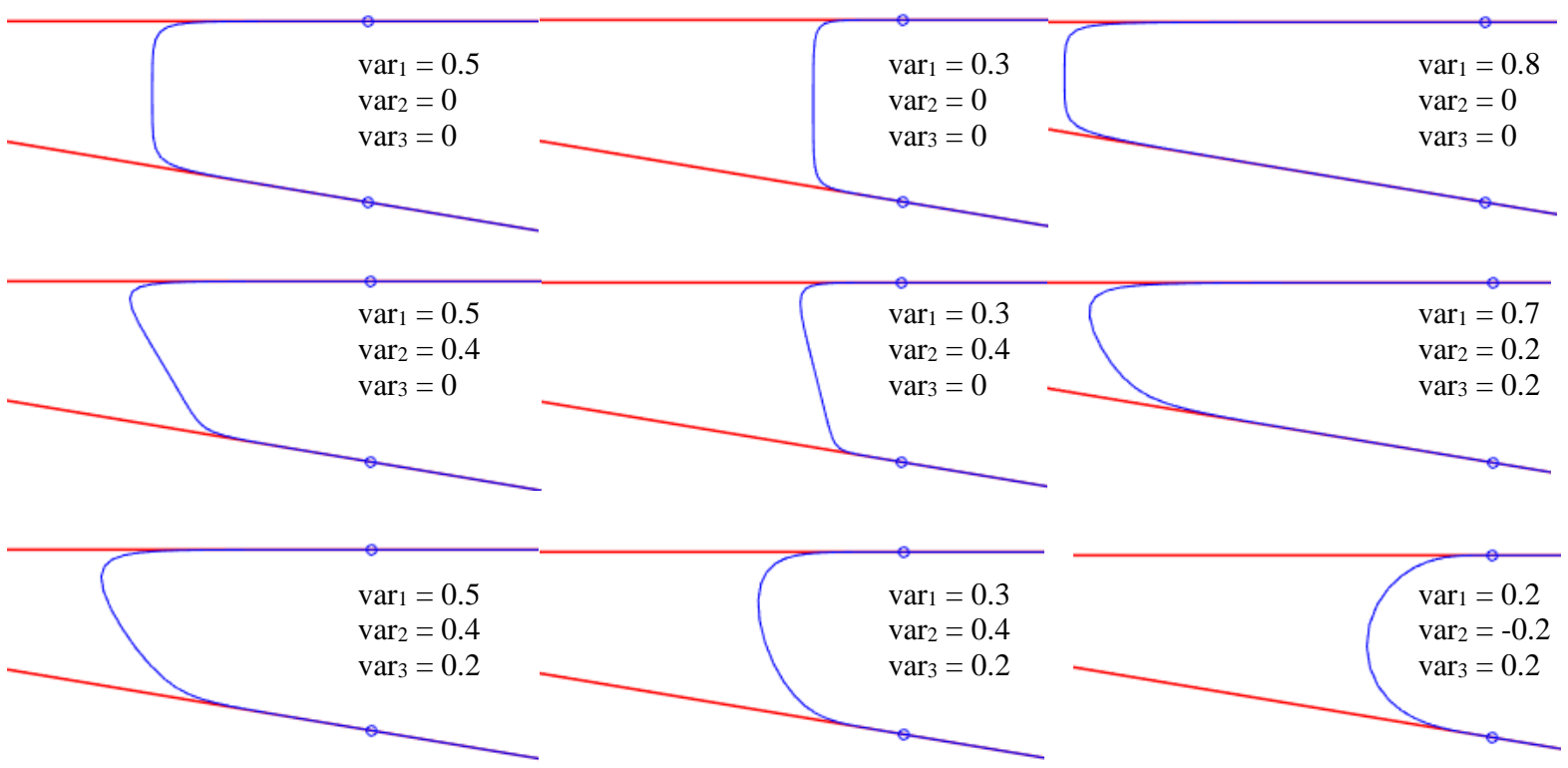

Figure 8. Leading edge shape examples of the fourth order rational Bézier parameterization. 
The fourth order RBLEs, when used on a wedge geometry consisting of straight lines, theoretically provide third order $\left(\mathrm{G}^{3}\right)$ geometric continuity at the interface, though in practice this is of limited significance in both geometrical and flow dynamics terms. Effectively though, it can provide an even smoother transition to zero curvature or infinite radius of curvature when compared with the cubic rational Bézier parameterization of Section B, as seen in Figure 9.
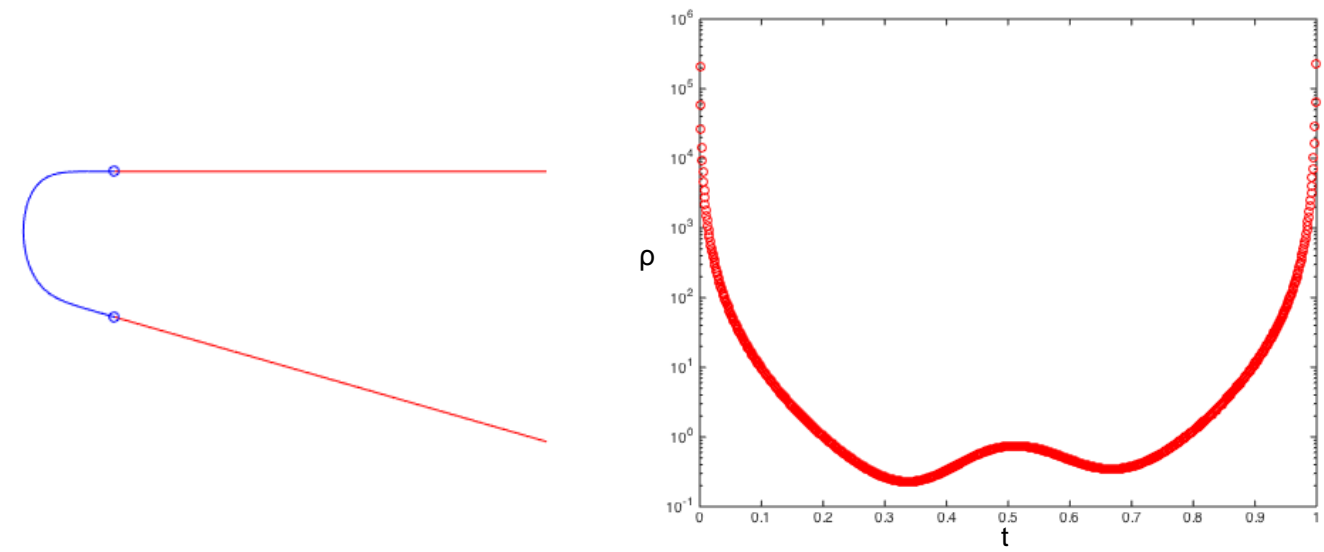

Figure 9. Radius of curvature across fourth order rational Bézier leading edge.

The variety of shapes obtained with this last approach is significantly wider, while we can also see that, even for a fixed value of the initial truncation length or thickness at the interface, the parameterization is able to drive itself to shapes of essentially lower thickness. It should be noted that because balancing the weights is more delicate in this case, it is harder to match finite radii of curvature at the end points as was done for the previous approach. It can be attempted by moving P2 accordingly, but careful reparameterization of the weights and design variables is required, something that will not be covered in this paper.

The extent to which it is beneficial to have this much control over the shape of the leading edge will be examined in more detail in the following sections. As far as manufacturability of such shapes goes, it is something that strongly depends on the scale of the bluntness needed. Finally, from now on each shape will be described only by the values of the design variables as they were defined in this section, and not the values of the weights.

\section{2D CFD Analysis of Shapes}

The ultimate goal of being able to look into a variety of different shapes for the blunt leading edges of hypersonic aircraft is to enable the design to get the best out of the tradeoff between the aerodynamic performance characteristics and the limits of the materials and thermal protection system. Blunter leading edges are required to cope with the increasing aerodynamic heating of higher velocities. As the bluntness increases so does the drag, while the lift can decrease due to the high-pressure flow at the underside of waverider shapes no longer being completely 'trapped', with the shock standing further away. The shape of the leading edge essentially affects the flowfield around the entire aircraft. As the bluntness-induced drag and peak temperatures at the leading edge are something that can be examined locally, we initially run a series of 2D CFD simulations to study the local aero-thermal properties of the shapes described earlier.

\section{A. Cold wall simulations.}

ANSYS Fluent ${ }^{11}$ was used for the simulations at this stage and a number of different case setups were considered. The first set of cases was set up with a cold wall $\left(\mathrm{T}_{\mathrm{w}}=300 \mathrm{~K}\right)$ and flow conditions matching Mach 8 flight at 100,000ft. While similar investigations were done for Mach 6 and varying dynamic pressures, the majority of the results presented here are for the Mach 8 case mentioned earlier unless otherwise stated. For all cases laminar flow was assumed around this part of the leading edge and therefore no turbulence model was used. The two values of interest were the drag and the peak heat flux. Grid independence was achieved with a 500x120 (along wall $\mathrm{x}$ wallnormal) initial structured C-type mesh, and three levels of adaptive mesh refinement in areas of high pressure gradient for better shock capturing. From this point we will consider a circular leading edge with radius $\mathrm{R}$ to be equivalent to the parametric shapes described earlier with thickness $2 \mathrm{R}$ for convenience, as a thickness cannot be 
defined the same way for circular leading edges due to the two interface points not being in the same streamwise position.

In Figure 10 we can see how the drag coefficient and peak heat flux vary for a $1 \mathrm{~cm}$ thick quadratic RBLE, for a range of 0.15 to 0.3 of the shape variable. The equivalent circular leading edge data point is also plotted. The wedge angle for this and most of the geometries that are presented was $9.5^{\circ}$, which is the deflection angle that results in a $\sim 15^{\circ}$ shock angle under the given flow conditions. All drag coefficient values are for the geometries extended downstream up to the point where the wedge thickness is $1.67 \mathrm{~cm}$.

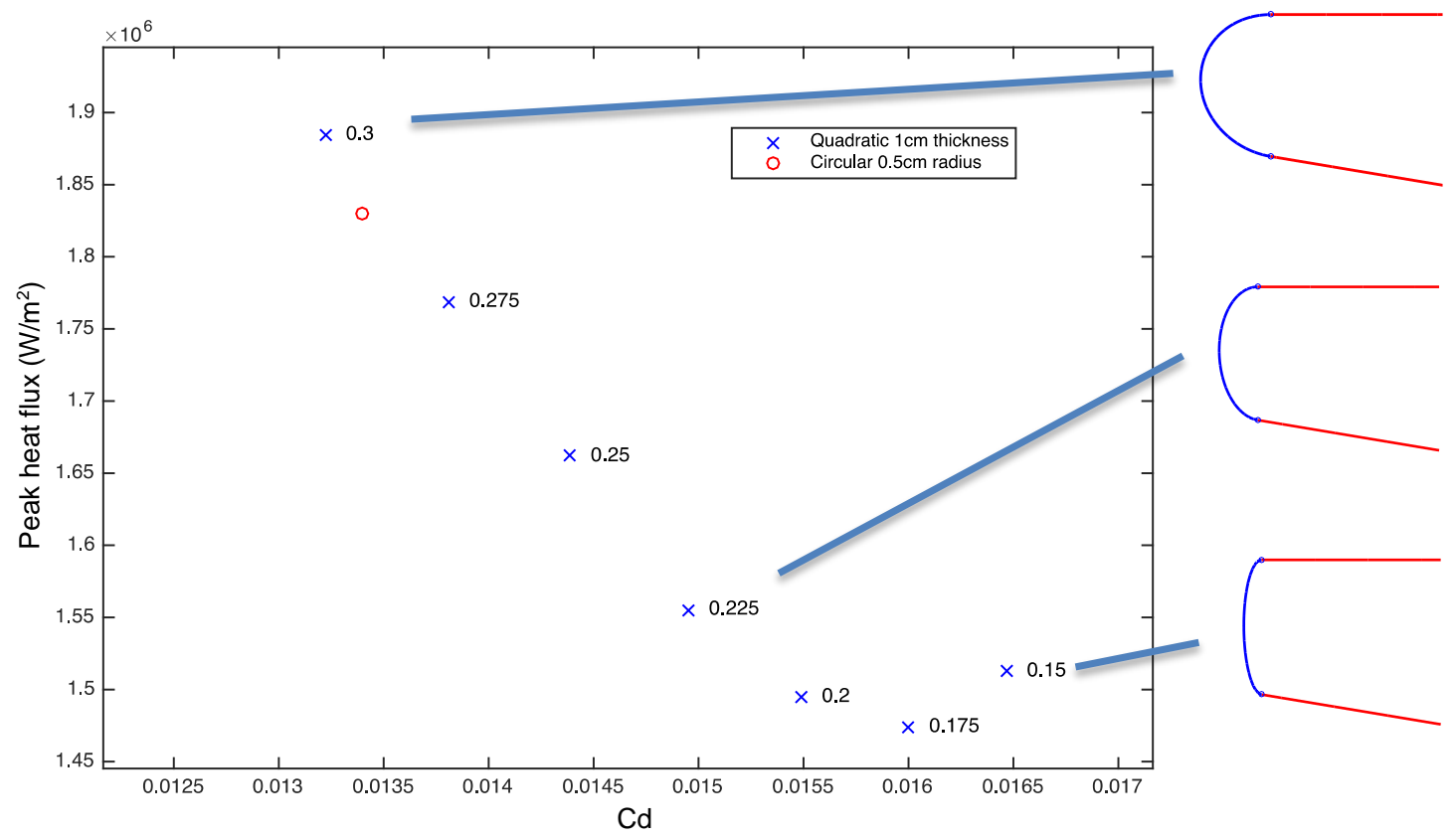

Figure 10. Drag coefficient and peak heat flux of a quadratic rational Bézier curve of $1 \mathrm{~cm}$ thickness, $T_{\mathrm{w}}=300 \mathrm{~K}, \mathrm{Minf}_{\mathrm{i}}=8, \mathrm{p}_{\mathrm{inf}}=1090 \mathrm{~Pa}$.

From Figure 10 we observe that, first of all, the parametric shapes can come close to the circular leading edge both in geometry and performance characteristics. As the geometry gets more blunt the drag increases while the peak heat flux at the stagnation region decreases and can reach values up to $20 \%$ lower than the equivalent circular leading edge. For geometries that become too blunt the heat flux around the sharply curved part close to the interface overcomes that of the stagnation point and the peak heat flux therefore increases again. The way to utilize these geometries if, for example, the initial goal was to replace the equivalent $0.5 \mathrm{~cm}$ radius circular leading edge is to adjust their thickness as well. This will enable the shape to have a lower drag coefficient for the same peak heat flux, something that will be demonstrated later on. It is also worth noting that, for cold wall cases of the same wall temperature, we observe the same pattern between the characteristics of the geometries for different flow conditions, something that was also observed by Rodi ${ }^{7-8}$. An example of the distribution obtained for a Mach 6, 75,000ft altitude case is shown in Figure 11.

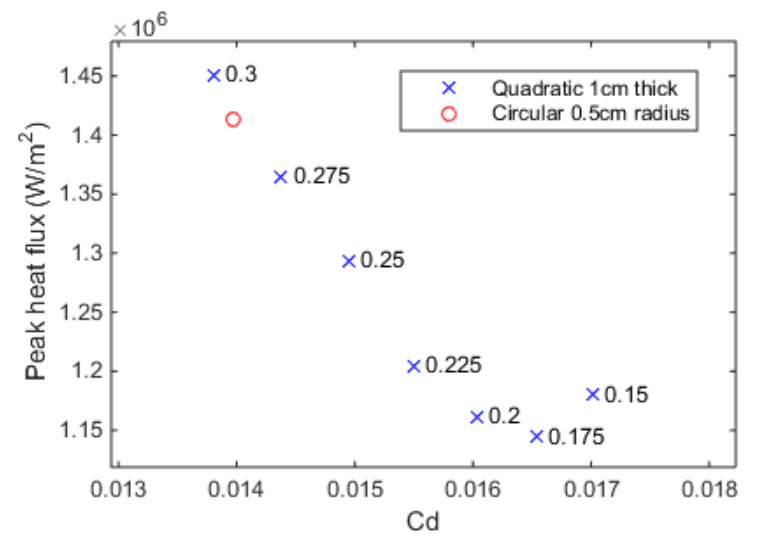

Figure 11. Drag coefficient and peak heat flux for $T_{w}=300 K, M_{\text {inf }}=6, p_{\text {inf }}=1090 P a$.

To more quickly obtain the sets of optimal geometries (Pareto front) of shapes controlled by multiple design variables such as the cubic and $4^{\text {th }}$ order RBLE parameterizations, meta-models were constructed using the kriging approach $^{12-13}$. The meta-models were based on a latin hypercube-obtained initial sample of 20 CFD calculations for the 2 design variable controlled cubic RBLEs and 40 for the 3 design variable controlled $4^{\text {th }}$ order RBLEs. 

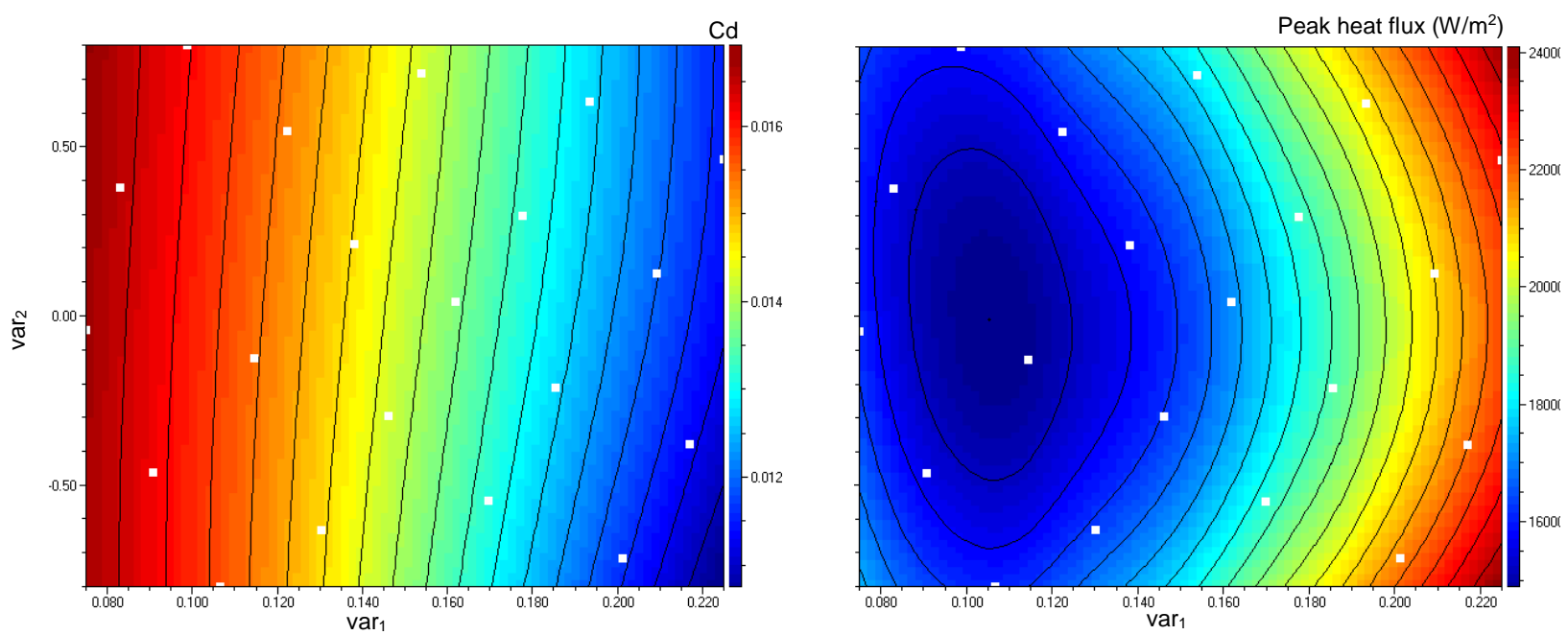

Figure 12. Contours of the kriging meta-model for drag and peak heat flux of the cubic RBLE with the initial sample as white squares, generated using QstatLab ${ }^{13}$.

The values obtained using the meta-model in this case are good approximations of the value of the CFD calculations although their accuracy can be further improved with more data points. Contours of the meta-models can be seen in Figure 12. Utilizing the kriging models we are then able to more densely populate the Pareto fronts of the 2 and 3 design variable controlled parameterizations using a brute force approach. The result can be seen in Figure 13.

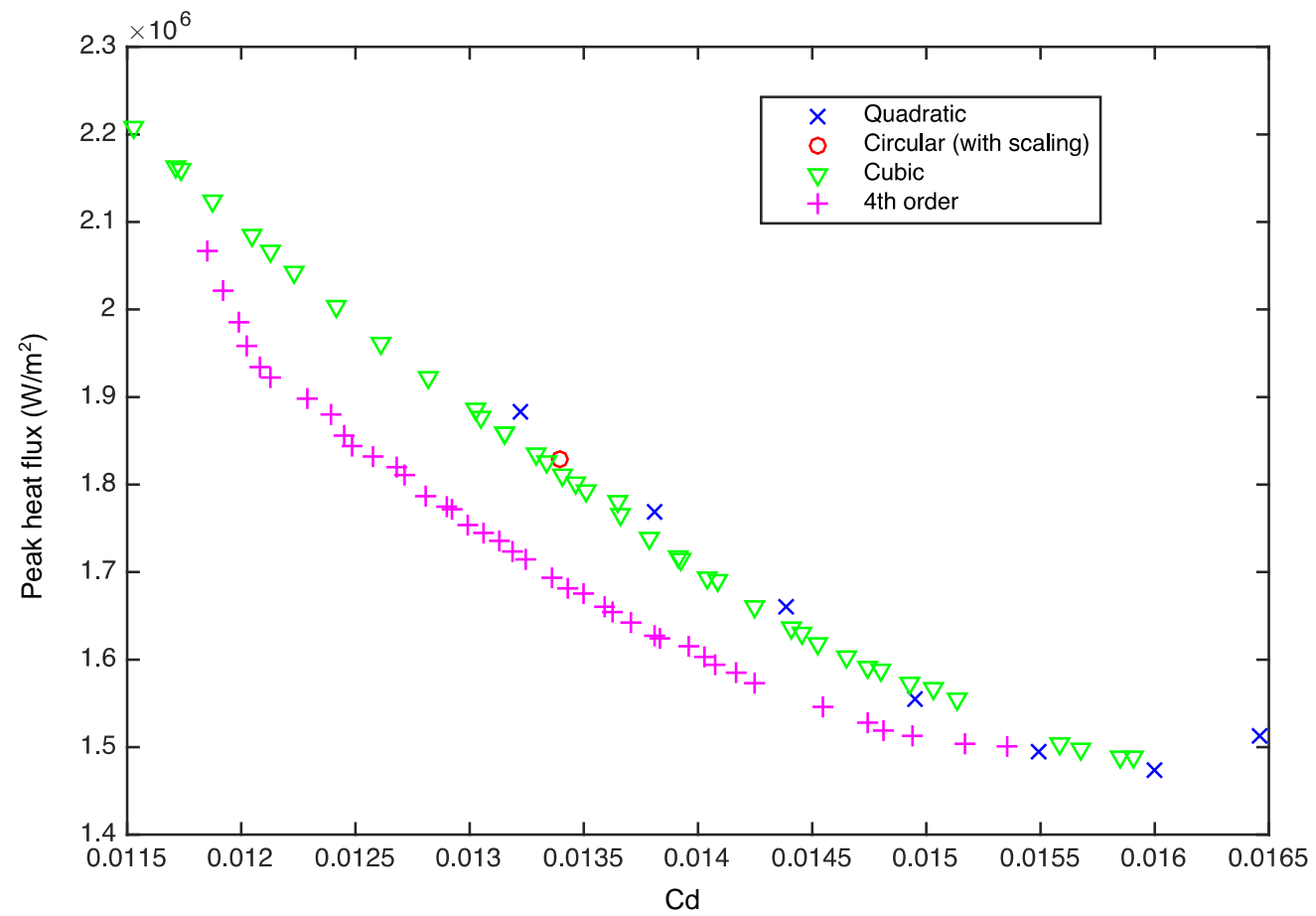

Figure 13. Sets of optimal solutions for the three different RBLE parameterizations, $T_{w}=300 \mathrm{~K}, M_{i n f}=8$, pinf $=1090$ Pa.

In Figure 13 we observe that while the solutions given by the more complex $4^{\text {th }}$ order RBLE shapes have a clear advantage over the quadratic ones, the cubic ones seem to be performing very similarly to the latter. Additionally, for the cubic RBLE all the optimal solutions seem to consist of a fixed value of the second design variable (that shifts the balance to the upper or lower part), while varying the other variable populates the Pareto front. Therefore, in case the extra design variable is fixed, the complexity of the parameterization would not be higher than the 
quadratic one, while, as was explained in Section II, it is a formulation that can provide $\mathrm{G}^{2}$ continuity of the geometry.

Now, to provide a more direct comparison between the parameterized shapes and the simple circular leading edge, we will compare the drag and peak heat flux of circular leading edges of varying radii with quadratic RBLE shapes of variable thickness as well. As in this case the quadratic RBLE is controlled with two design variables, the thickness and one shape parameter $\left(\operatorname{var}_{1}\right)$, a similar use of meta-models was followed. The set of optimal solutions can be seen in Figure 14, where it is also compared with circular leading edges. It is worth noting that the Pareto front was dominated by geometries whose shape parameter had a value of around 0.182 with only the thickness varying along the distribution. What we also see in Figure 14, is that a circular leading edge with a radius of $0.5 \mathrm{~cm}$ can be replaced with a thinner quadratic RBLE with the same peak heat flux that will have a drag coefficient around $20 \%$ lower. This RBLE would be $\sim 30 \%$ thinner, i.e. $0.7 \mathrm{~cm}$ thick in this case. Finally, in Figure 15 we see a comparison of the heat flux distribution around the blunt part of the geometry of the quadratic RBLE, with $\operatorname{var}_{1}=0.182$, that dominates the aforementioned Pareto front and the equivalent circular leading edge.

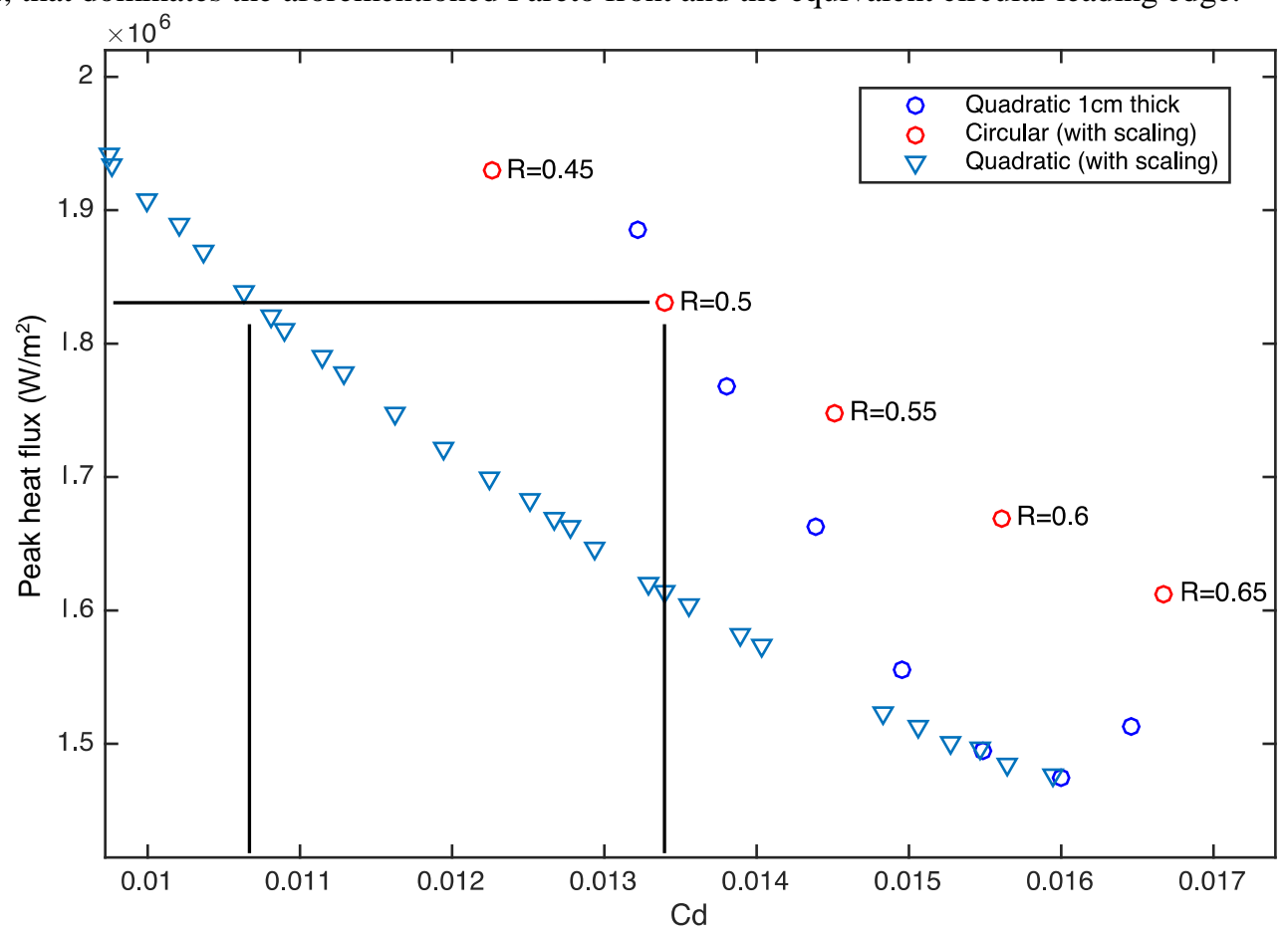

Figure 14. Circular leading edge and quadratic $R B L E$ comparison, $T_{w}=300 K, M_{i n f}=8, p_{i n f}=1090 P a$.

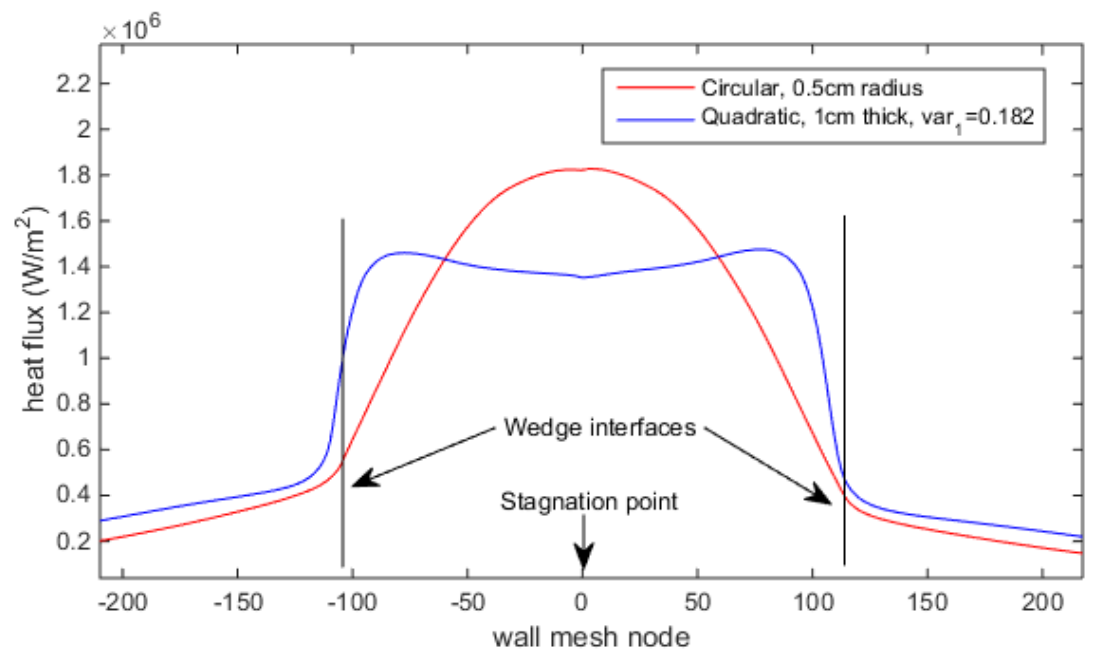

Figure 15. Heat flux distribution for the dominant quadratic RBLE and the equivalent circular leading edge, $T_{\mathrm{w}}=300 \mathrm{~K}, \mathrm{M}_{\mathrm{inf}}=8, \mathrm{p}_{\mathrm{inf}}=1090 \mathrm{~Pa}$.

10

American Institute of Aeronautics and Astronautics 


\section{B. Equilibrium temperature simulations.}

The next series of simulations were set up to calculate equilibrium temperatures along the wall, using conditions that are more representative of the operation of a thermal protection system intended for hypersonic cruising. A first set of cases was run with a radiative equilibrium temperature condition at the wall, and a second set with conductive heat transfer within the solid leading edge tip as well. The flow conditions in the results presented were again set to match Mach 8 flight at 100,000ft. The emissivity of the wall is set to 0.9 and any radiation originating from the hot gases around the geometry is neglected, so only the wall radiates energy. For the second set of cases a relatively high thermal conductivity of $200 \mathrm{~W} / \mathrm{mK}$ was assumed for the heat conduction calculations within the solid leading edge. The values of interest are now the drag coefficient and the peak wall temperature.

The results that follow are for a set of quadratic RBLE shapes for values of the design variable $\left(\operatorname{var}_{1}\right)$ ranging from 0.15 to 0.325 and a thickness of $1 \mathrm{~cm}$, also compared with the equivalent $(0.5 \mathrm{~cm}$ radius) circular leading edge. In Figure 16 we can see the drag coefficient and peak temperatures that were calculated for the different shapes for both case setups.

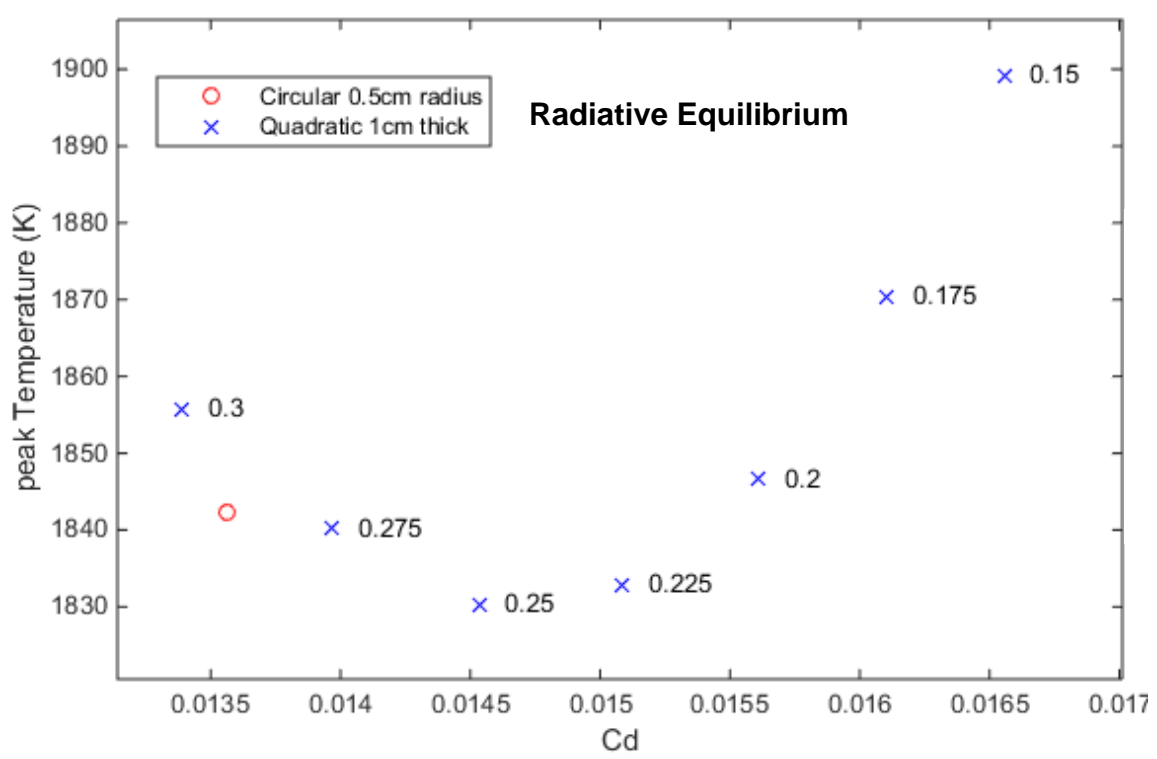

Quadratic RBLE shapes

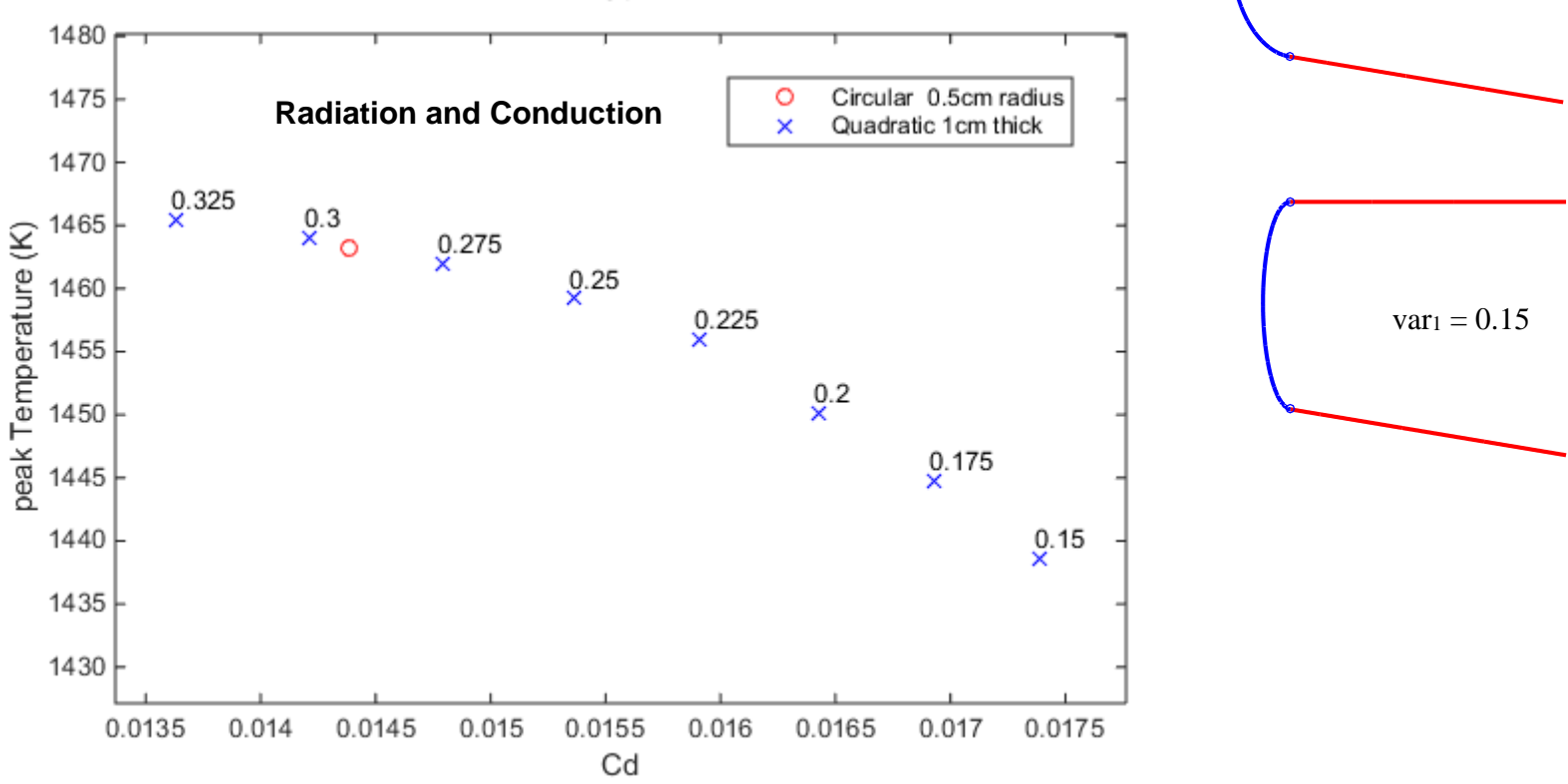

Figure 16. Peak temperature and drag coefficient for radiative equilibrium temperature conditions (top) and radiative and convective equilibrium conditions (bottom) for different values of var $_{1}, \varepsilon=0.9, \mathrm{k}=200 \mathrm{~W} / \mathrm{mK}$, $M_{\text {inf }}=8, p_{\text {inf }}=1090$ Pa. 
A first observation is that the distributions shift significantly when radiation and thermal conduction is accounted for, while different distributions were also observed for different values of the thermal conductivity of the leading edge material. Even for the radiative equilibrium temperature set of cases, that are equivalent to a case with 0 thermal conductivity, the shape that shows the lowest peak temperature is different from the one where the lowest peak heat flux was observed (see Figure 10). Moreover, as expected, the range of temperatures around the leading edge becomes narrower with increasing thermal conductivity, something that significantly drives down the peak temperatures, illustrated in Figure 17.

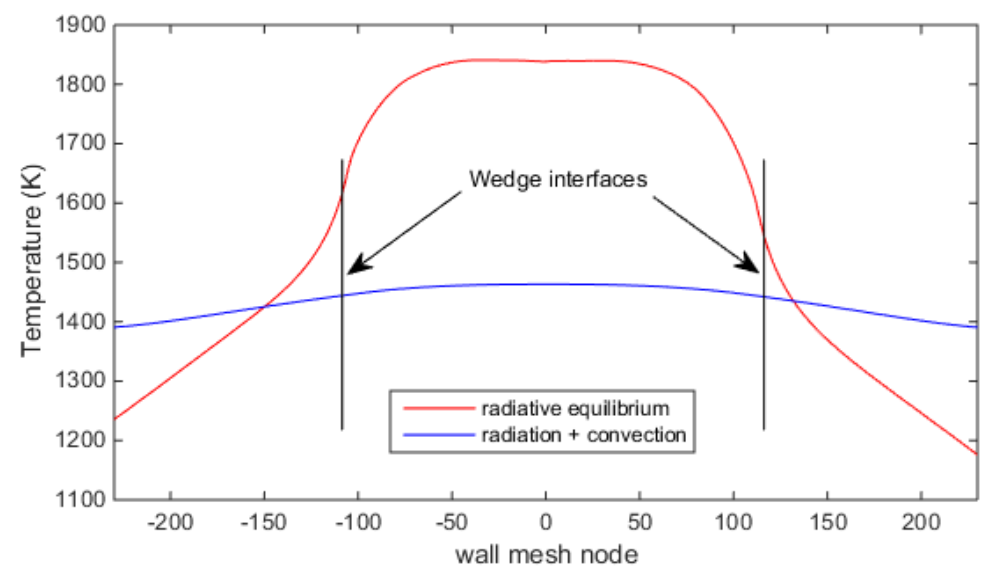

Figure 17. Temperature distributions around circular leading edge for radiative equilibrium conditions and radiation with heat conduction, $\varepsilon=0.9, \mathrm{k}=200 \mathrm{~W} / \mathrm{mK}, M_{\text {inf }}=8, p_{\text {inf }}=1090 \mathrm{~Pa}$.
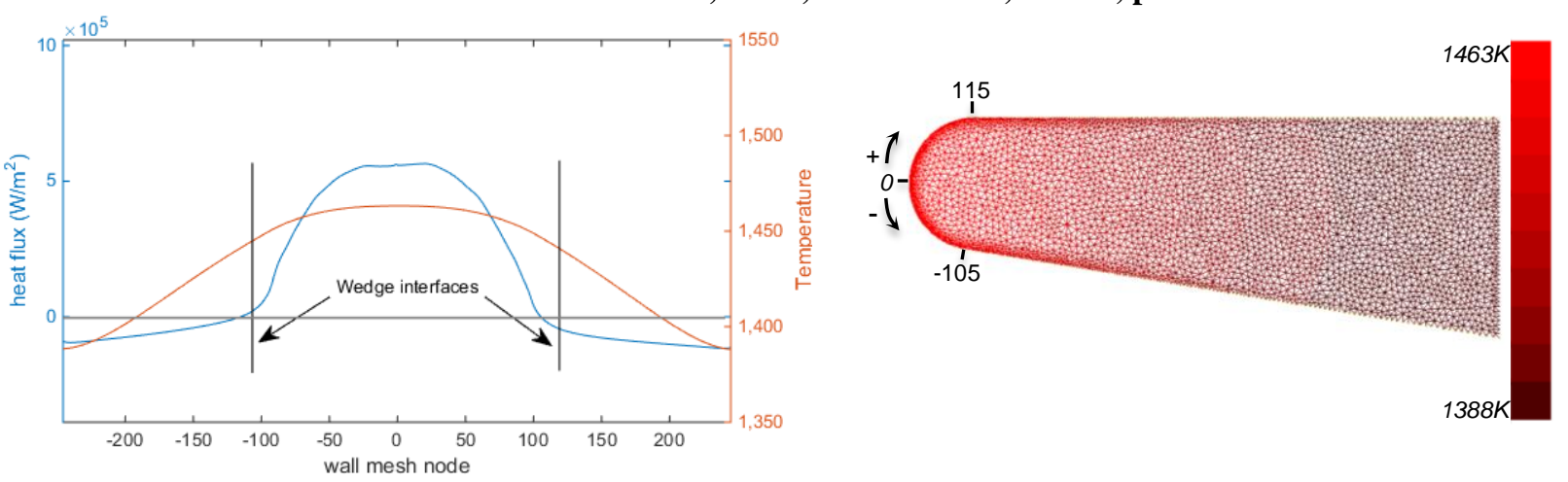

Figure 18. Heat flux and temperature around circular leading edge (left) and temperature distribution within the solid (right, mesh visible), $\varepsilon=0.9, \mathrm{k}=200 \mathrm{~W} / \mathrm{mK}, \mathrm{M}_{\mathrm{inf}}=8$, $\mathrm{p}_{\mathrm{inf}}=1090 \mathrm{~Pa}$.

Apart from being interesting from an aerodynamics perspective, these observations indicate that the optimal shapes strongly depend on the specific conditions of each case, with the differences in the distributions seen in Figures 16 and 10 supporting that. To elaborate, even if for cold wall conditions a Mach number independence has been observed when it comes to the optimum shapes, it is the nature (active, passive etc.) and specific characteristics of the thermal protection system that will direct any design process on a case by case basis. That is where such geometry models, parameterized with low numbers of design variables and able to achieve a wide range of meaningful shapes, assist in making higher fidelity and multi-physics design studies affordable.

\section{Receptivity - Transition Effects}

The boundary layer turbulent transition can have a profound effect on a hypersonic vehicle's design and performance. With the leading edge being where the boundary layer starts developing, even small changes in its shape can potentially affect the transition mechanism. In this section we present some preliminary results of investigations into the effect of using a geometry with increased geometric continuity at the wedge interface. A numerical simulation, aimed at studying the leading edge receptivity process to fast acoustic waves, was carried out using a fourth-order accurate direct numerical simulation code. This was for a hypersonic flow at Mach 7.3 over a $20^{\circ}$ half-angle blunt wedge, where the $0.1 \mathrm{~mm}$ circular nose radius has been replaced with a fourth order rational Bézier curve. This leading edge shape, as described in Section II, provides second order geometric continuity of the 
wall geometry at the wedge junction. The specific geometry was designed to be roughly close to the geometry of a circular leading edge in the area around the stagnation point, attempting to keep an almost constant radius of curvature in that section, and a smoother transition to infinite radius of curvature towards the interface with the wedge, as seen in Figure 20. The main reason for this is that the investigation is intended to study the effect of the increased geometric continuity and not just a different geometry.

The acoustic waves have been inserted in the domain with 10 frequencies ranging from $50 \mathrm{kHz}$ to $500 \mathrm{kHz}$, with random phase, and constant amplitude equal to 1.0E-04 at each frequency (with reference to the freestream density fluctuation amplitude). The receptivity results are compared with those obtained for the case of a cylinder-wedge with circular leading edge and nose radius of $0.1 \mathrm{~mm}$ with the same flow conditions (shown in the work of Cerminara and Sandham ${ }^{15}$, presented at the $45^{\text {th }}$ AIAA Fluid Dynamics Conference, Dallas, June 2015). In particular, a direct comparison is provided for the pressure and heat flux fluctuation amplitude spectra at three different positions along the wedge. Table 1 shows the flow conditions for both the cases.

Table 1. Flow conditions of the numerical simulations

\begin{tabular}{|c|c|c|c|c|c|}
\hline $\mathrm{M}_{\text {inf }}$ & Unit Re $(1 / \mathrm{m})$ & $\mathrm{T}_{0}(\mathrm{~K})$ & $\mathrm{T}_{\text {inf }}(\mathrm{K})$ & $\mathrm{p}_{\text {inf }}(\mathrm{Pa})$ & $\mathrm{T}_{\mathrm{w}} / \mathrm{T}_{\text {inf }}$ \\
\hline 7.3 & $4.4 \times 10^{6}$ & 2740 & 234.034 & 2004.301 & 1.273 \\
\hline
\end{tabular}

The following figure shows a sketch of the two different leading edge geometries in non-dimensional coordinates.

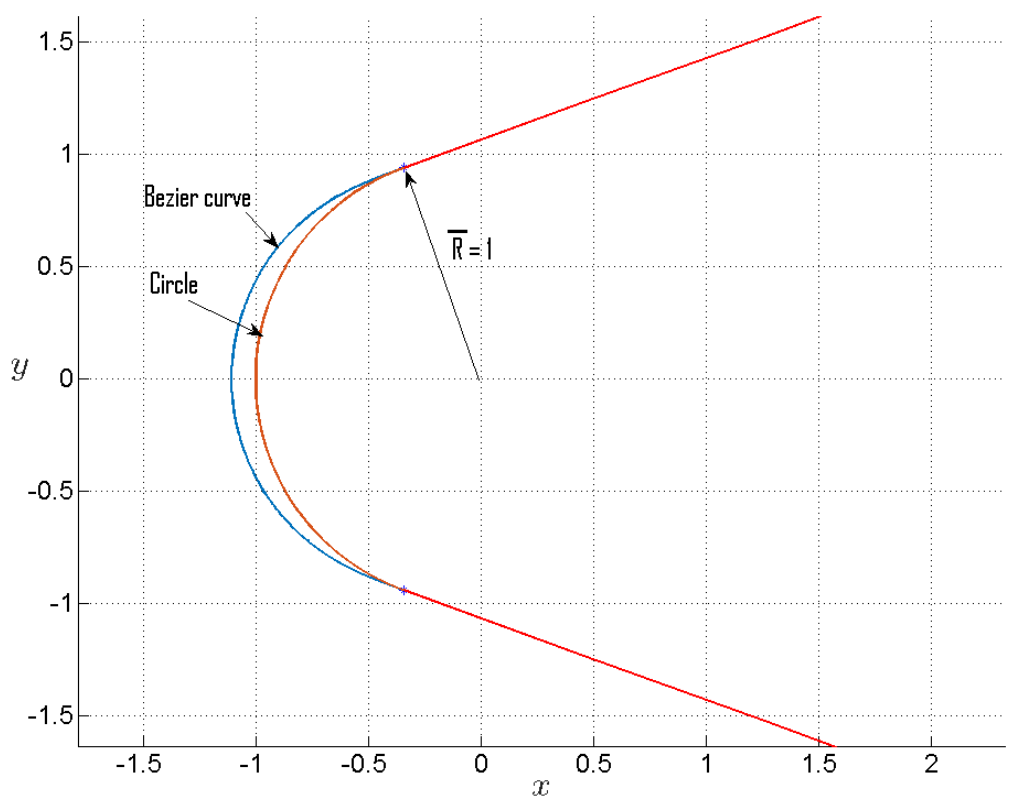

Figure 19. Sketch of the two leading edge geometries in non-dimensional coordinates: circle (red line), and rational Bézier curve (blue line). The radius of the circle $(R)$ is the characteristic length scale for both the geometries.

As can be seen in Figure 19, the length scales are normalised with respect to the radius, R, of the circle (which in the case of the Bézier curve represents the distance between the wedge junction point and the origin of the coordinate system), so that in the numerical simulations the Reynolds number, computed using the nose radius of the circle $\left(\operatorname{Re}=\left(\rho_{\text {inf }} \mathrm{U}_{\text {inf }} R\right) / \mu_{\text {inf }}\right.$, where $\rho_{\text {inf }}, U_{\text {inf }}$ and $\mu_{\text {inf }}$ are the freestream density, velocity and dynamic viscosity of the flow respectively), is the same for both the cases and equal to 440. The following figure shows the variation of the radius of curvature along the wall for the two geometries, between the two wedge-junction points. 


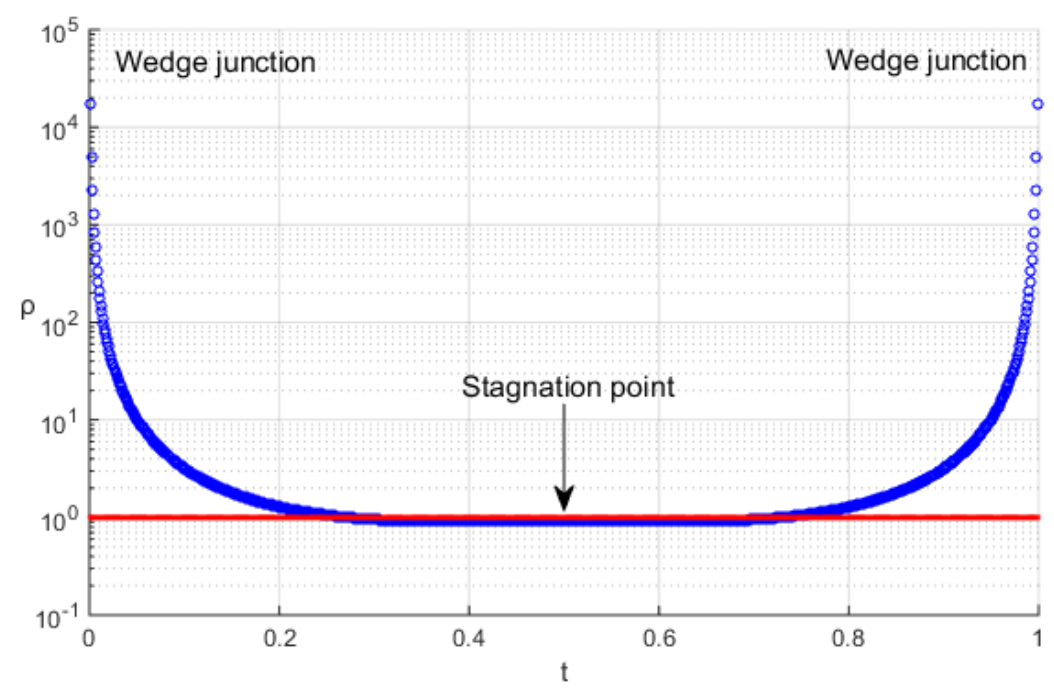

Figure 20. Radius of curvature at different positions along the leading edge curves: circle (red line), Bézier curve (blue line).

Figure 20 highlights the difference in radius of curvature between the two leading edge curves. In particular, in the case of a circular leading edge the radius of curvature keeps constant (red line) and equal to 1 (in nondimensional coordinates) along the curvilinear part of the wall, while becoming equal to infinity on the wedge, thus resulting in a curvature discontinuity at the circle-wedge junction. In contrast, the rational Bézier curve leading edge shape is characterised by a radius of curvature (indicated by a blue line in Figure 20) that gradually varies from a value of 0.95 at the area around the stagnation point to infinity at the interface with the wedge, thus providing curvature continuity at the wedge junction. The rational Bézier curve-based leading edge results in a slightly more slender body, with the radius of curvature at the stagnation point $5 \%$ lower than that of the circular leading edge.

Figure 21 shows a comparison of the results for the mean wall pressure in the wedge junction region between the two cases with different leading edge geometries (the $x$ variable in Figure 21 is the non-dimensional coordinate along the body symmetry axis in the Cartesian reference system, while the pressure $\mathrm{p}_{\mathrm{w}}$ is normalised with the freestream reference value $\left.\rho_{\mathrm{inf}} \mathrm{U}_{\mathrm{inf}}^{2}\right)$. As is evident in the figure, the wall pressure profile for the rational Bézier curve leading edge has a smoother gradient at the wedge interface than the case of circular leading edge shape, due to the curvature continuity.

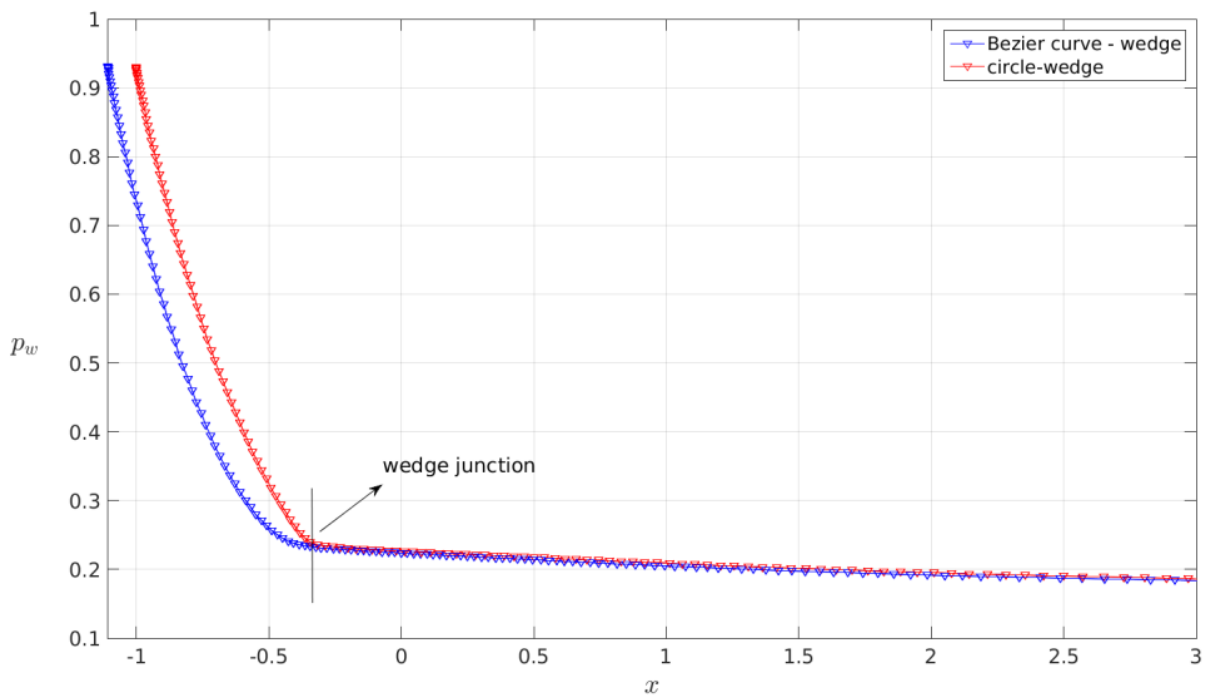

Figure 21. Mean pressure profiles along the wall (stagnation point of RBLE shape is $\sim 0.1 \mathrm{R}$ upstream of the circular one).

14

American Institute of Aeronautics and Astronautics 
Figure 22 shows the results for the density fluctuation field obtained by imposing fast acoustic waves in the freestream, for the case of a blunt wedge with rational a Bézier curve leading edge, and the length of the body extending up to about $400 \mathrm{R}$ downstream.

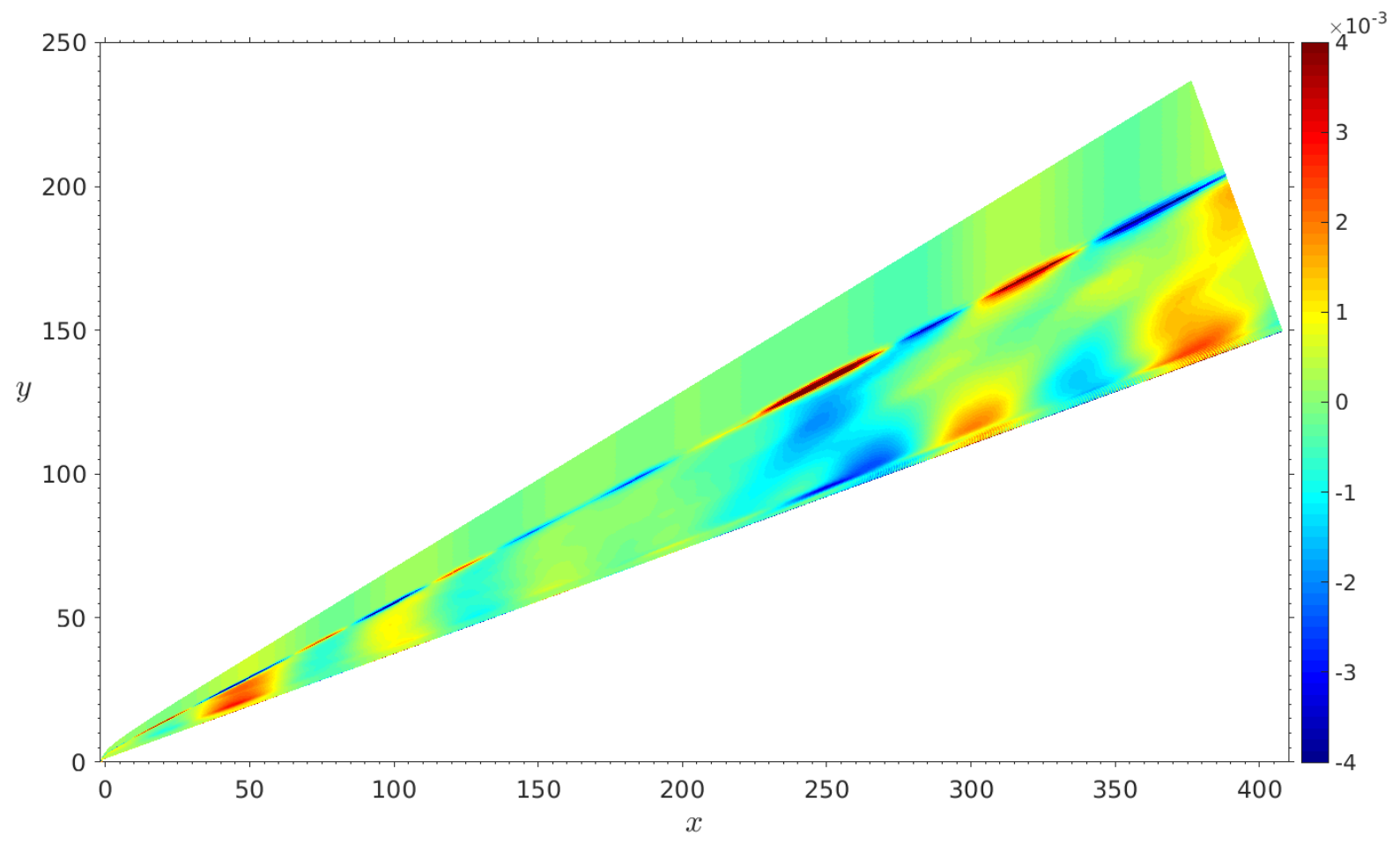

Figure 22. Instantaneous density fluctuation field ( $\left.\rho / \rho_{\text {inf }}\right)$.

The density fluctuation field shows that the wave structure behind the shock consists in general of a series of two fluctuation peaks of the same sign (one located in the boundary layer and the other just behind the shock) travelling downstream (this trend is evident in particular in the last portion of the wedge, for $x>250$ ). This pattern is qualitatively in good agreement with results found in literature (e.g. Kara et al. ${ }^{16}$ ), and shows the presence of boundary-layer flow structures developing downstream.

Figure 23 shows a comparison between the two cases at three different points along the wedge, corresponding to the $x$ non-dimensional coordinate values $x=297.3, x=319.4$ and $x=347.5$. Here, the pressure and heat flux fluctuation amplitude spectra, computed with a Fast Fourier Transform approach, are presented on the three different positions on the wall. In particular, the pressure fluctuation amplitudes are given at all three positions, while the heat flux fluctuation amplitude spectrum is given only at the position $x=347.5$ (see Cerminara and Sandham ${ }^{15}$ for more details). Note that the wall pressure fluctuation amplitude $\left(\mathrm{p}^{\prime}{ }_{\mathrm{w}}\right)$ is normalised with the freestream pressure fluctuation amplitude $\left(\mathrm{p}^{\prime}{ }_{\text {inf }}\right)$, while the heat flux fluctuation amplitude $\left(\mathrm{q}^{\prime}{ }_{\mathrm{w}}\right)$ is normalised with the freestream reference quantity for the energy flux $\left(\rho_{\mathrm{inf}} \mathrm{U}_{\mathrm{inf}}{ }^{3}\right)$. 

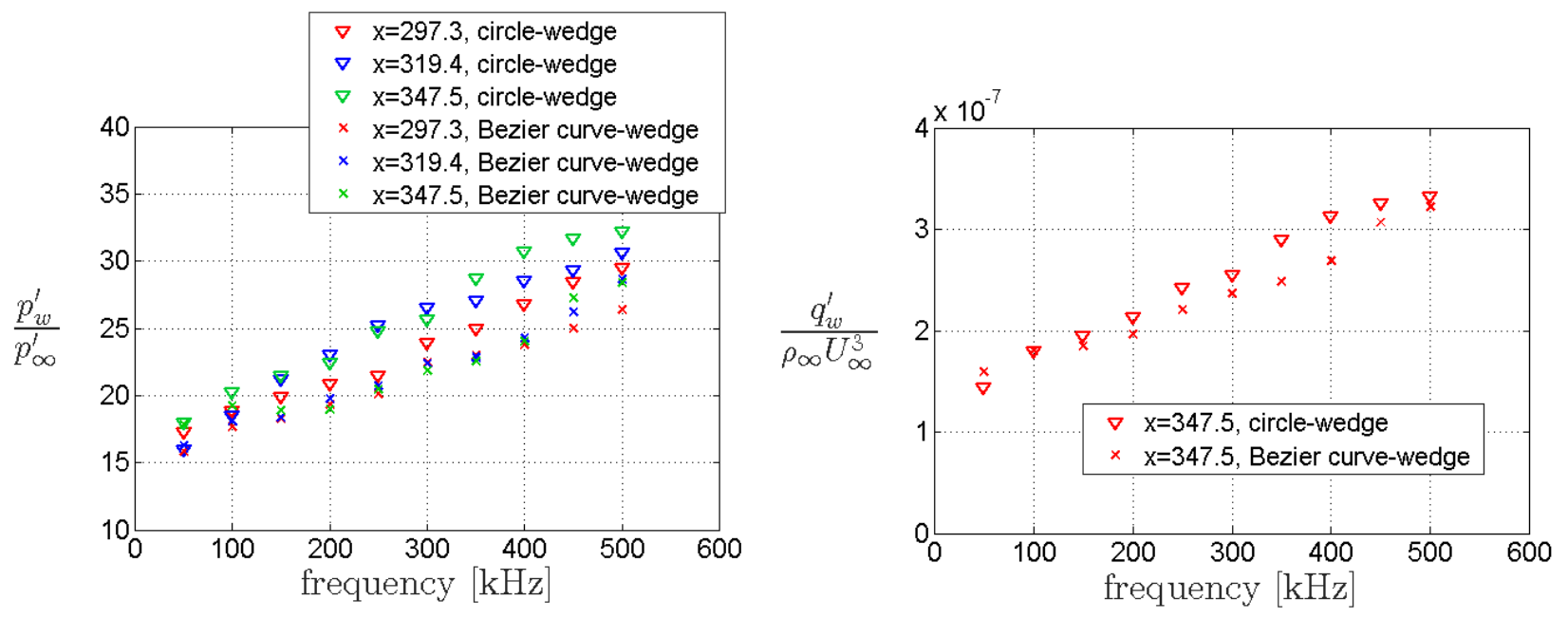

\section{Figure 23. Pressure (left) and heat flux (right) fluctuation amplitude spectra at three different points along the wall.}

The results in Figure 23 reveal that the fluctuation trend at the different frequencies is qualitatively similar between the two cases, for both the pressure and the heat flux, with the fluctuation amplitude increasing at increasing frequencies. However, in the case of the rational Bézier curve leading edge the receptivity is in general lower than the case with a circular leading edge. This difference in fluctuation amplitude between the two leading edge geometries seems, in turn, to increase with the frequency and with the $x$ distance along the wall, for both pressure and heat flux spectra, up to a maximum reached around $350 \mathrm{kHz}-400 \mathrm{kHz}$. In particular, the maximum difference is obtained at $400 \mathrm{kHz}$ and $x=347.5$ (where the pressure and heat flux fluctuation amplitudes for the circle-wedge case are respectively about $20 \%$ and $14 \%$ higher than for the rational Bézier curve leading edge case).

The difference in the receptivity levels between the two different leading edge geometries may be due in general to the slightly stronger shock formed in front of the circular leading edge (with higher radius of curvature at the stagnation point), which produces a higher amplification of the acoustic waves behind the shock in the nose region, and hence higher wall pressure fluctuation levels transmitted downstream. However, part of this difference may be also a consequence of the curvature continuity in the case of the rational Bézier leading edge, which guarantees a gradual variation of the wall pressure at the wedge junction (as shown in Figure 21), and might produce differences in the mechanism through which the external waves are internalised into the boundary layer.

In conclusion, from the results shown above, the fourth order rational Bézier leading edge geometry that provides $\mathrm{G}^{2}$ continuity, seems to have a slightly stabilizing effect on the wall receptivity to fast acoustic waves, in particular at the higher frequencies. However, in order to quantify the effect of the different shock curvature and strength due to the different stagnation point radius, and the effect of the curvature continuity, further investigation is needed. Moreover, the study needs to be complemented with the receptivity to slow acoustic waves, which lead to the generation of the dominant unstable mode inside the boundary layer in hypersonic flows, as evident by results available in the literature (e.g. Kara et al. ${ }^{16}$, Malik and Balakumar ${ }^{17}$ ).

\section{Integration on 3D Waverider Geometries}

There are a number of different approaches when it comes to integrating the $2 \mathrm{D}$ geometry formulations described earlier on 3D waverider forebody geometries. In this last section we will present our preferred method and provide some general remarks on the subject as evaluating and quantifying the merits of each different approach requires complex and computationally expensive case studies.

It is common to truncate the sharp leading edge geometry perpendicular to the leading edge in order to accommodate the bluntness. There are, however, some benefits in truncating the geometry and positioning each RBLE section along the osculating planes in the case of an osculating cones/osculating flowfield generated waverider forebody or along the planes on which the streamlines were traced in axisymmetric or wedge shock-based inverse design methods. In this way, maintaining geometric continuity at the interface of the blunt leading edge shape with the waverider's upper and lower surface becomes more straightforward, especially when $2^{\text {nd }}$ order 
geometric continuity (which can be achieved with the parameterization described in this work) is desirable. Moreover, even if the original geometry was not $\mathrm{G}^{2}$ continuous in the spanwise direction due, for example, to the shape of the leading edge, $\mathrm{G}^{2}$ continuity will be maintained along the general direction of the streamlines (were general direction hints at the fact that bluntness in swept parts will generate cross-flow to adjacent osculating planes). Both approaches are illustrated in Figure 24. To obtain the desired thickness when truncating the geometry with the second method (II), the truncation length has to be adjusted according to the local sweep angle and inclination of the osculating planes when truncating along them.

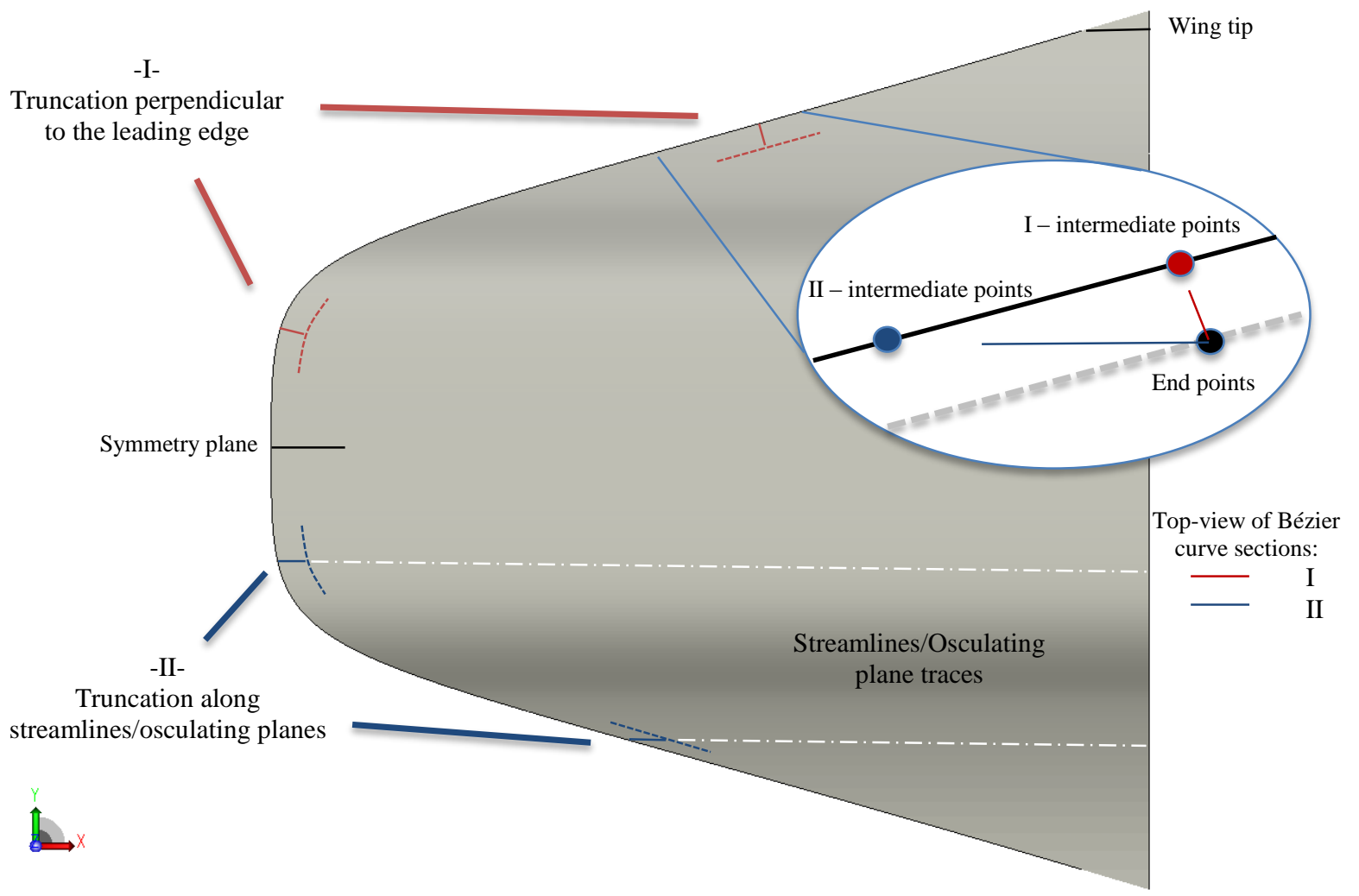

Figure 24. Top view of a sharp leading edge waverider forebody with illustrations of truncation options to accommodate the bluntness.

At this stage, apart from the thickness and shape of each section, it is also meaningful to control how those characteristics are distributed along the leading edge, from the symmetry plane at the front to the wing tip. While the 2D evaluations will be valid for the symmetry plane section, swept sections are subject to reduced aerodynamic heating. More specifically, the stagnation point heating of circular swept leading edges can be related to the nonswept one using the empirical equation ${ }^{18}$ :

$$
\frac{q_{\text {swept }}}{q_{\text {non-swept }}}=(\cos \lambda)^{1.1}
$$

Therefore, in order to optimally utilize the thermal protection system's limits, the thickness can be reduced as the local sweep increases. The relation for the heating rate of two circular leading edges with different radii is ${ }^{19}$ :

$$
\frac{q_{R 1}}{q_{R 2}}=\sqrt{\frac{R 2}{R 1}}
$$

Using these empirical relations and substituting the radius with the thickness of the RBLE shapes, we can distribute the thickness across the leading edge in order to obtain roughly the same peak heating rate as the 
symmetry section. This will result in lower drag while utilizing the thermal protection system to its limits across the entire leading edge. The same approach was also followed in Ref. 8.

$$
d_{\text {swept }}=d_{\text {symmetry }}(\cos \lambda)^{2.2}
$$

When this method is directly applied to geometries that have areas along the leading edge with sudden variations in sweep angle, the planform shape of the original geometry can be significantly altered. Areas where the leading edge is almost non-swept and the sweep starts increasing rapidly are most sensitive. Figure 25 includes an example of this, with the sweep starting to increase significantly around $y=0.2$. The resulting geometry ends up with two 'bumps' that are faced head on with the oncoming flow while their thickness is much lower than the nominal zerosweep thickness that was defined.
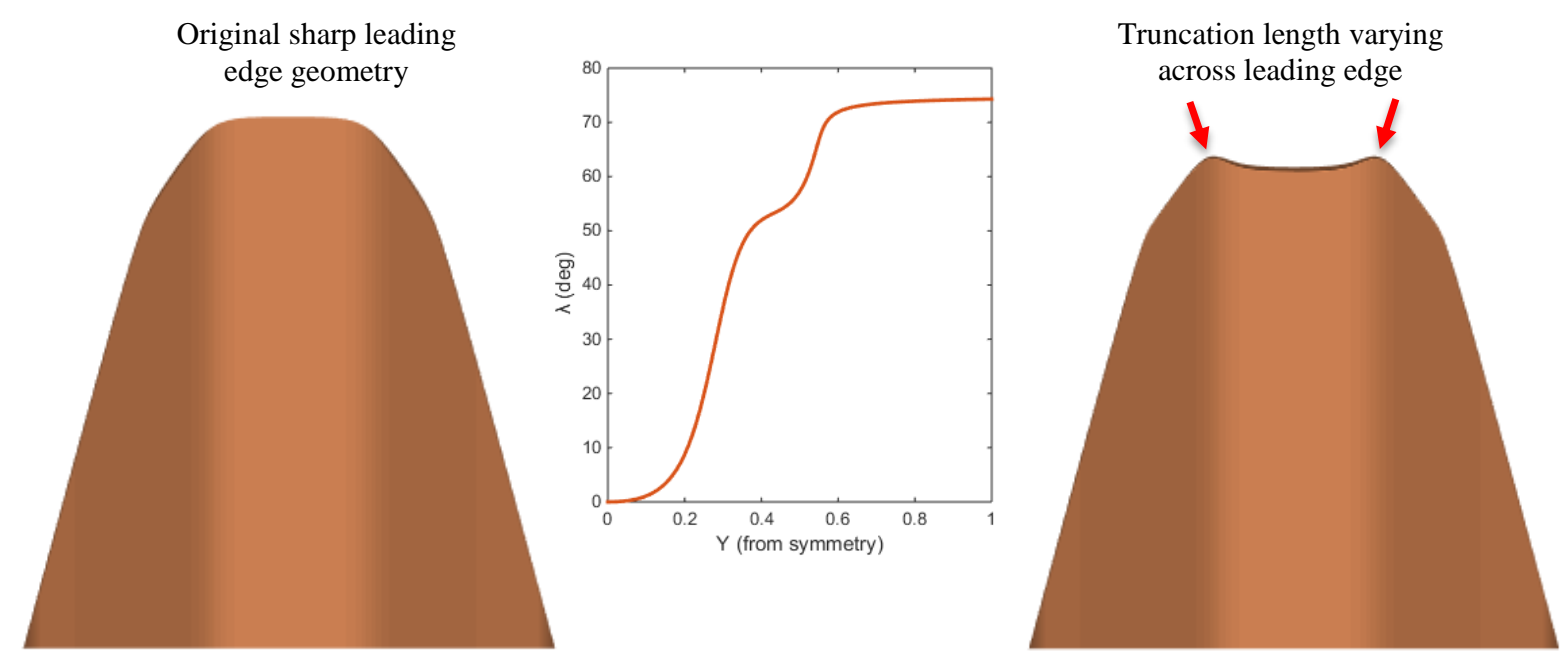

Figure 25. Planform deformation when varying truncation length across leading edge according to original geometry's sweep.

To counter this issue at the front of the geometry we can apply a limiter that will not allow the geometry to be truncated less than what is needed in order prevent it from protruding in front of the sections before it, as we move from the symmetry plane to the wing tip. The result of applying this limiter can be seen in Figure 26, together with the modified truncation distribution.

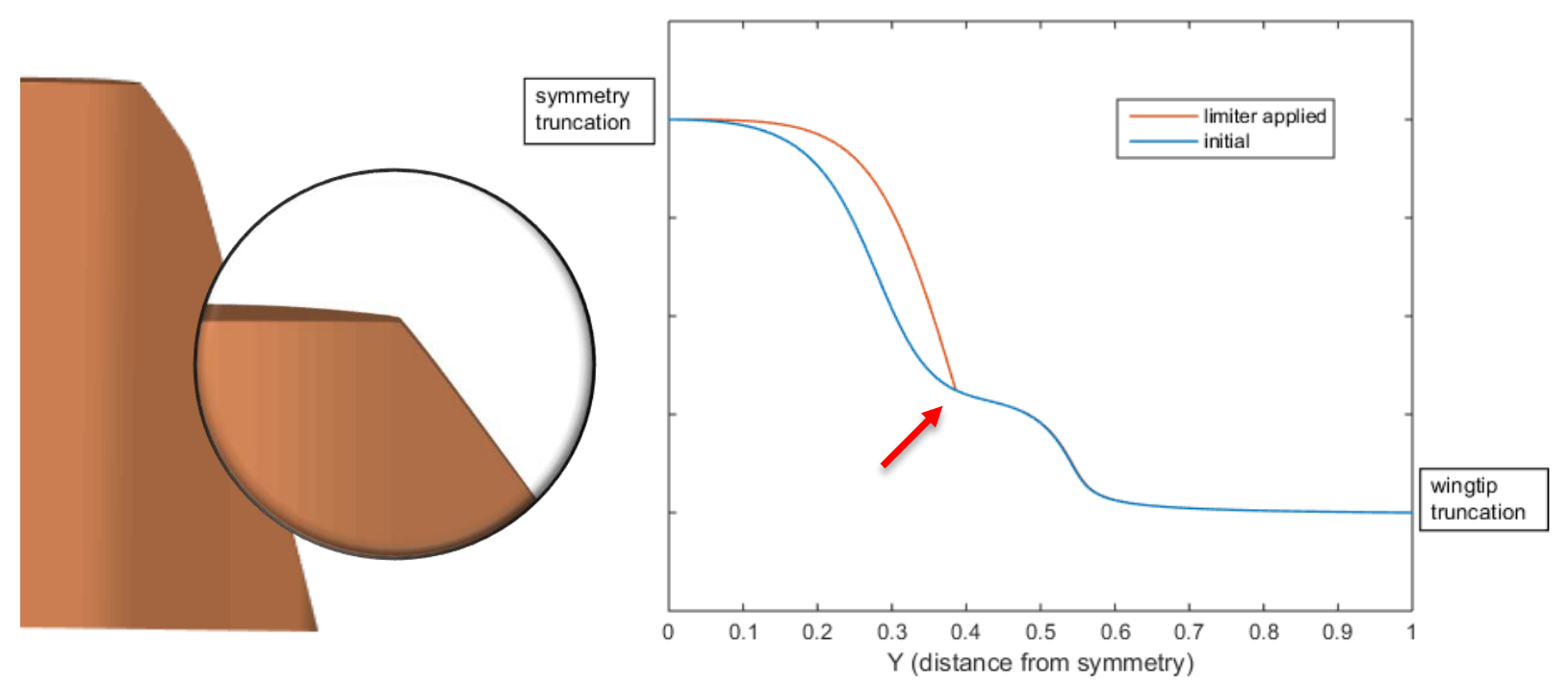

Figure 26. Truncation length distribution and resulting geometry with limiter applied. 
The limiter does solve the previous issue, however it creates a sharp edge at the point where it switches off. To remedy this, a smoothing of the truncation length distribution in that area can be performed, using, for example, a locally weighted scatterplot smoothing method. The results of this can be seen in Figure 27.

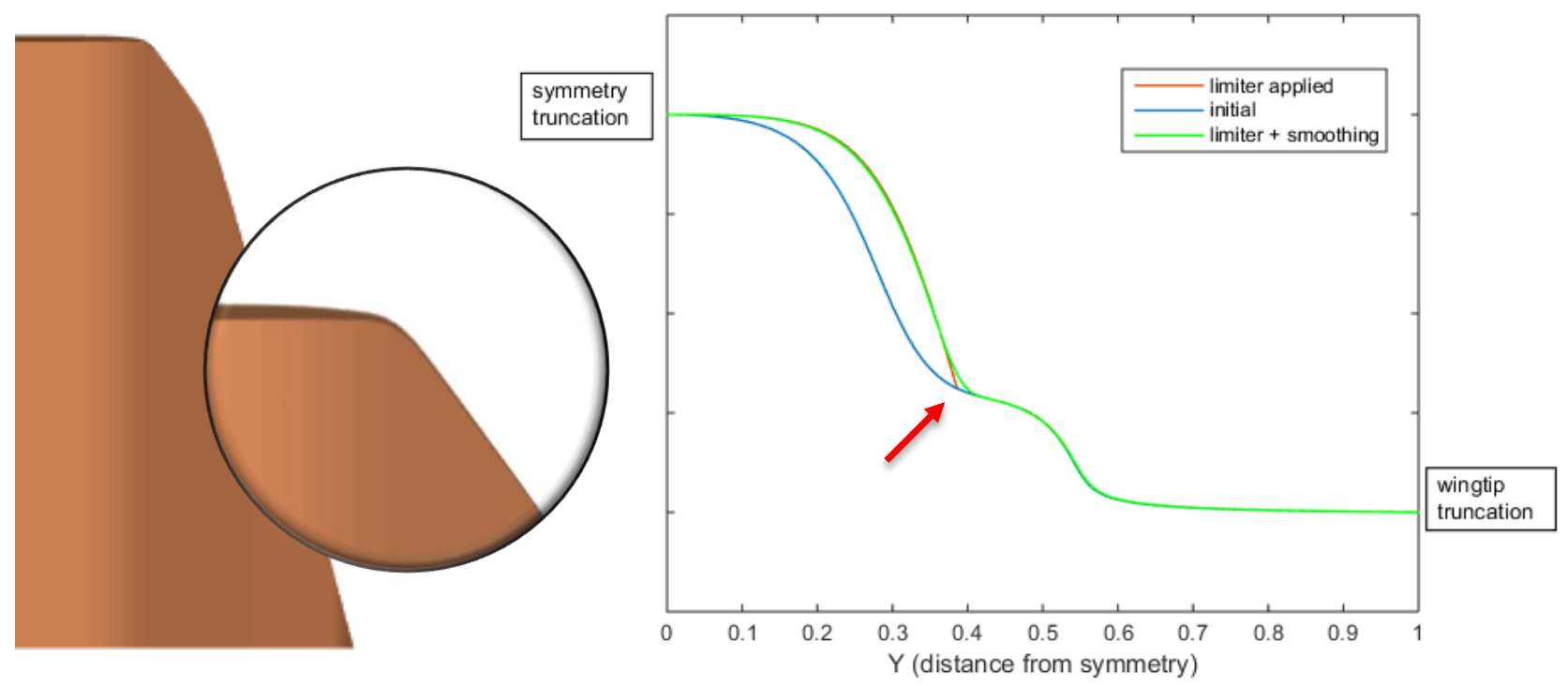

Figure 27. Truncation length distribution and resulting geometry with limiter and smoothing applied.

An alternative to the latter is running an iterative method that will keep correcting the thickness distribution as the planform shape of the geometry and effective sweep changes, until a converged shape is reached. Additionally, instead of truncating the geometry it can also be advantageous to raise the upper surface in order to accommodate the bluntness of the leading edge, as has been suggested in previous studies ${ }^{20}$. This displacement can also be blended with the truncation, as seen in Figure 28, something that leads to a variety of additional options. Although this introduces an additional design parameter for each section that also has to be distributed along the leading edge, it also opens up a number of possibilities. For example, it is now possible to generate the required thickness for the blunt leading edge without affecting

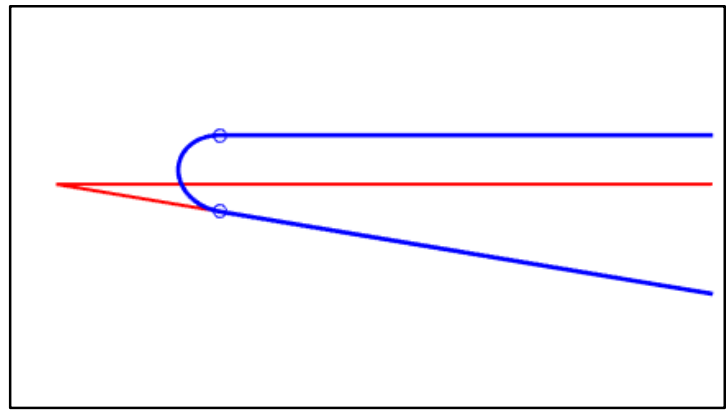

Figure 28. Blended displacement of upper surface and truncation of original geometry to accommodate the bluntness. the planform shape of the waverider by manipulating the truncation and displacement in order to keep the tip of the geometry at each section in the same place. What also needs to be considered is that the equations described earlier do not take into account any effects that the variations of sweep along the leading edge can have on aerodynamic heating, as they generally apply for straight, constant sweep, leading edge segments. It is, therefore, expected that the optimal leading edge geometries can only be reached utilizing controlled distributions. The previous analysis does however provide a reasonable basis for a self-designing geometry, which avoids the use of one or two more design variables that would have to control that custom distribution.

Finally, it is worth noting that apart from the blunt leading edge's thickness, it can also be beneficial to vary the shape parameters that control the RBLE geometries across the leading edge. Aerodynamic heating considerations apart, this can potentially further limit any losses in lift due to leakage of the high pressure flow from the underside of the waverider around the leading edges as well. Additionally, we may find that for swept leading edges we obtain a different set of optimal shapes when it comes to drag and peak heating rates or temperatures. To quantify any potential advantages though, a series of full 3D CFD simulations will be required. This, together with the development of more detailed geometry parameterization schemes, will be the topic of future research activity. 


\section{Conclusions}

An efficient and robust approach to designing blunt leading edges for waverider forebodies has been developed and investigated. The parameterization is very robust and can provide a variety of meaningful shapes. The limited number of variables that control the shape make it well-suited for use in large-scale design optimization studies of full waverider geometries. The shapes are also able to achieve second order $\left(\mathrm{G}^{2}\right)$ geometric continuity at the interface with the truncated original waverider geometry. Some preliminary results indicate that this can potentially affect the receptivity and turbulent transition mechanisms. The geometries can, depending on the case, demonstrate significantly better performance than circular leading edges, as shown in Section III-A. This, among other factors, strongly depends on the scale and thickness of the blunt part needed. If, for example, a bluntness radius of only a few millimeters is required, the ease of manufacturing would potentially outweigh the benefits of using a more sophisticated shape. Finally, we presented our preferred method and some remarks for integrating the parametric shapes on 3D waverider forebodies, with 3D flow effects being an area that can be further investigated.

\section{Acknowledgments}

The authors would like to acknowledge the financial support provided by MBDA UK Ltd and Innovate UK: the work reported herein has been undertaken as part of GHandI (TSB 101372), a UK Aerospace Technology Institute project.

\section{References}

${ }^{1}$ Eggers, A.J., Ashley, H., Springer, G.S., Bowles, J.V., and Ardema, M.D., "Hypersonic Waverider Configuration from the 1950's to the 1990's", AIAA Paper 93-0774, 31st Aerospace Sciences Meeting, Reno, NV, 1993.

${ }^{2}$ Bowcutt, K.G., Anderson, J.D., Capriotti, D., "Viscous Optimized Hypersonic Waveriders”, AIAA Paper 87-0272, 24th Aerospace Sciences Meeting, Reno, NV, 1987.

${ }^{3}$ Sobieczky, H., Dougherty, F., and Jones, K.D., "Hypersonic Waverider Design from Given Shock Waves", First International Hypersonic Waverider Symposium, University of Maryland, College Park, MD, 1990.

${ }^{4}$ Rodi, P.E., "The Osculating Flowfield Method of Waverider Geometry Generation", AIAA Paper 2005-0511, 44th AIAA Aerospace Sciences Meeting, Reno, NV, 2005.

${ }^{5}$ O’Brien, T.F., Lewis, M.J., "Power Law Shapes for Leading-Edge Blunting with Minimal Shock Standoff", AIAA Journal of Spacecraft and Rockets, Vol., 36, No. 5, September-October 1999, pp. 653-658.

${ }^{6}$ Santos, W.F.N., and Lewis, M.J., "Aerothermodynamic Performance Analysis of Hypersonic Flow on Power Law Leading Edges," AIAA Journal of Spacecraft and Rockets, Vol. 42, No. 4, July-August 2005, pp588-597.

${ }^{7}$ Rodi, P.E., "Optimization of Bezier Curves for High Speed Leading Edge Geometries", AIAA Paper 2013-1004, January 2013.

${ }^{8}$ Rodi, P.E., "Integration of Optimized Leading Edge Geometries onto Waverider Configurations", AIAA Paper 2015-1700, January 2015.

${ }^{9}$ Kontogiannis, K., Taylor, N., Sóbester, A., “On the Conceptual Design of Waverider Forebody Geometries”, AIAA Paper 2015-1009, 53 ${ }^{\text {rd }}$ AIAA Aerospace Sciences Meeting, Kissimmee, FL, 2015.

${ }^{10}$ Sederberg, T. W., "Computer Aided Geometric Design Course Notes”, BYU, 2012.

${ }^{11}$ ANSYS FLUENT, Release 14.0, ANSYS, Inc.

${ }^{12}$ Jones, D.R., Schonlau, M. and Welch W.J., "Efficient global optimization of expensive black-box functions", Journal of Global Optimization, 13, 455-492, 1998.

${ }^{13}$ QstatLab, v6, Available at: http://qstatlab.co.uk.

${ }^{14}$ Anderson, J. D., "Hypersonic and High Temperature Gas Dynamics", Second Edition, AIAA Education Series, 2006.

${ }^{15}$ Cerminara, A., Sandham, N., D., "Leading-edge Receptivity to Acoustic Waves for High-Speed Flows over a Blunt Wedge", AIAA Paper 2015-3078, 45th Fluid Dynamics Conference, Dallas, TX, 2015.

${ }^{16}$ Kara, K., Balakumar, P., and Kandil, O. A., "Receptivity of Hypersonic Boundary Layers Due to Acoustic Disturbances over Blunt Cone", AIAA Paper 2007-945, 2007.

${ }^{17}$ Malik, M. R., and Balakumar, P., "Acoustic receptivity of Mach 4.5 boundary layer with leading-edge bluntness", Theoretical and Computational Fluid Dynamics, 21 (5), pp. 323-342, 2007.

${ }^{18}$ Hayes, J.R., Neumann, R.D., "Introduction to the Aerodynamic Heating Analysis of Supersonic Missiles", AIAA Progress in Astronautics and Astronautics, Volume 142, Mendenhall M.R. (ed.), 1992.

${ }^{19}$ Bertin, J.J., "Hypersonic Aerothermodynamics", AIAA Education Series, New York, New York, 1994.

${ }^{20}$ Tincher, D., Burnett, D., "Hypersonic Waverider Test Vehicle: A Logical Next Step", Journal of Spacecraft and Rockets, 31, 392-399, 1994. 